NIS'IIR 4442

\title{
User's Guide to CMMAP: Cement
}

Microstructure Modelling and

\section{Analysis Package}

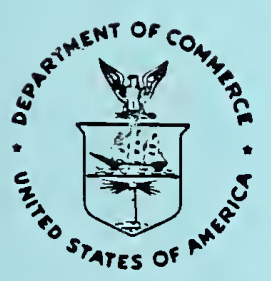

U.S. Department of Commerce

National Institute of Standards and Technology

Center for Building Technology

Gaithersburg, MD 20899 



\section{User's Guide to CMMAP: Cement}

\section{Microstructure Modelling and}

\section{Analysis Package}

Dale P. Bentz

Edward J. Garboczi

October 1990

U.S. Department of Commerce

Robert A. Mosbacher, Secretary

National Institute of Standards and Technology

John W. Lyons, Director

Center for Building Technology

Gaithersburg, MD 20899 



\begin{abstract}
A collection of modelling and analysis programs dealing with cement microstructure has been assembled into a single package. This manual describes the custom user interface, CMMAP, which allows the user to access and execute the assembled software. Specific programs exist to create starting microstructures, simulate hydration, and analyze both starting and hydrated microstructures. Available analyses include evaluation of diffusion coefficients, simulation of mercury intrusion porosimetry, and assessment of the connectivity of phases within a cement microstructure. The menu-based interface program is described in detail and several examples are presented which demonstrate the capabilities of CMMAP.
\end{abstract}

Keywords: Cement, computer imaging, connectivity, diffusion, hydration, microstructure, modelling, simulation. 
ABSTRACT $\ldots \ldots \ldots \ldots \ldots \ldots \ldots \ldots \ldots \ldots \ldots \ldots \ldots \ldots \ldots \ldots \ldots \ldots$

LIST OF FIGURES $\ldots \ldots \ldots \ldots \ldots \ldots \ldots \ldots \ldots \ldots \ldots \ldots$

LIST OF TABLES $\ldots \ldots \ldots \ldots \ldots \ldots \ldots \ldots \ldots \ldots \ldots \ldots$

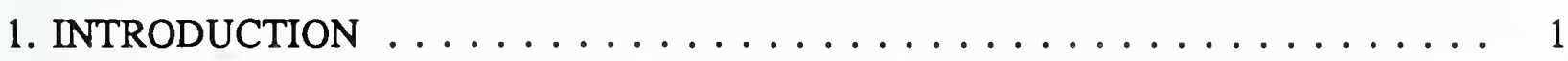

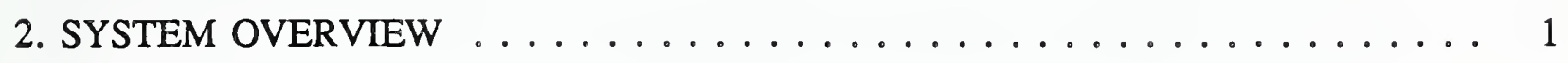

3. MODELLING AND ANALYSIS PROGRAMS $\ldots \ldots \ldots \ldots \ldots$

3.1 Generate a Starting Microstructure . . . . . . . . . . . . 3

3.2 Run a Hydration Model . . . . . . . . . . . . . . . . . . 5

3.3 Compute Diffusivities . . . . . . . . . . . . . . . . . 7

3.4 Perform Simulated Mercury Intrusion $\ldots \ldots \ldots \ldots \ldots . \ldots \ldots$

3.5 Compute Connectivity of Pore Space . . . . . . . . . . . . . . . . . . 10

3.6 Perform General Microstructural Characterization . . . . . . . . . . . 11

3.7 Perform Utility Functions $\ldots \ldots \ldots \ldots . \ldots \ldots . \ldots \ldots$

3.7.1 Specialty PIXAR Programs . . . . . . . . . . . . . 12

4. USER INTERFACE . . . . . . . . . . . . . . . . . 13

5. EXAMPLE SESSIONS AND OUTPUT $\ldots \ldots \ldots \ldots \ldots \ldots \ldots \ldots \ldots$

6. ACKNOWLEDGEMENT ........................... 25

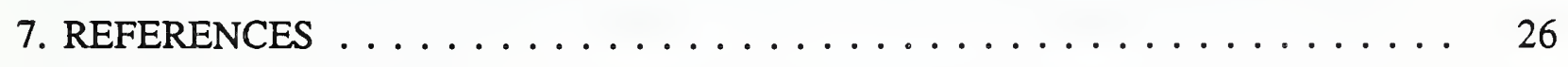

APPENDIX A START UP PROCEDURES $\ldots \ldots \ldots \ldots \ldots \ldots \ldots \ldots$

APPENDIX B USEFUL UNIX COMMANDS . . . . . . . . . . . . . . . 28

APPENDIX C USEFUL PIXAR COMMANDS . . . . . . . . . . . . . . . . 29

APPENDIX D BIBLIOGRAPHY . . . . . . . . . . . . . . . 30

APPENDIX E EQUATIONS RELEVANT TO CMMAP . . . . . . . . . . 31

APPENDIX F EXAMPLE SESSION TRANSCRIPTS . . . . . . . . . . 33 


\section{LIST OF FIGURES}

Figure 1. Graphical View of Coordinate System of PIXAR Screen .......... 2

Figure 2. Starting 3-D Microstructure Consisting of Randomly Parked Spheres Shown as 100 Slices, Each 100*100 Pixels ............... 4

Figure 3. Two-Dimensional Mercury Intrusion Produced by CMMAP . . . . . . . 9

Figure 4. Starting 2-D Microstructure Consisting of Randomly Parked Monosize Circles ......................... 14

Figure 5. Fig. 4 after Intrusion by Mercury with Meniscus Radius Set at Five Pixels or a Meniscus Diameter of Eleven Pixels . . . . . . . . . 16

Figure 6. Fig. 4 after Intrusion by Mercury with Meniscus Radius Set at Eight Pixels or a Meniscus Diameter of Seventeen Pixels

Figure 7. Results of Blind Ant Diffusivity Analysis of the 3-D Microstructure Shown in Fig. 2 ............................ 18

Figure 8. Image of One of the 100 Slices of the 3-D Microstructure Shown in Fig. 2 after 20 Cycles of Hydration by CMMAP ... . . . . . . 20

Figure 9. Starting 2-D Microstructure Consisting of Randomly Parked Cement Particles

Figure 10. 2-D Microstructure of Fig. 9 after Four Cycles of Hydration by

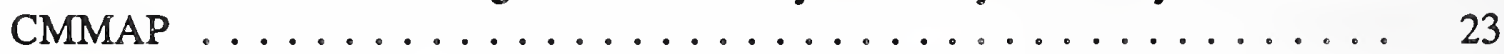

Figure 11. Black and White Image Showing Porosity Isolated from Fig. $10 \ldots 24$

Figure 12. Autocorrelation Curve Determined for Porosity Shown in Fig. 11 . . . . . 24

\section{LIST OF TABLES}

Table I. Summary of 3-D Hydration Results . . . . . . . . . . . . . . . . . 19

Table II. Summary of $2-\mathrm{D}$ Hydration Results . . . . . . . . . . . . . . 22 


\section{INTRODUCTION}

This manual describes models for cementitious materials developed and acquired during the last few years by the Building Materials Division of the Center for Building Technology for execution on the division's UNIX-based SUN/PIXAR computer imaging workstation. The models are all digital image-based. Space (two-dimensional area or three-dimensional volume) is represented as arrays of discrete elements, called pixels. Each pixel is uniquely identified as one material phase for example pore space, hydration product, or intruding mercury. The models provide the capabilities for creating an original cement microstructure consisting of cement particles mixed with water, hydrating the original microstructure for a given number of cycles using user-specified hydration parameters, and characterizing original and hydrated microstructures by a variety of measures such as diffusivity, pore connectivity, and particle size distributions.

The novice user of the system does not need to understand the inner workings of these models to make use of them. To such a user, the models should appear as black boxes which accept a well-defined input, execute a process, and produce a well-defined output. Admittedly, at times, users may be interested in investigating the models under "white box" conditions where the inner workings of the models should be understood. An example of this would be if the user wanted to modify the hydration rules and monitor their effect on developing microstructure. This situation, however, is viewed as the exception to the rule. Thus, to make the system more accessible to the novice user, a custom user interface has been developed.

The documentation in this manual serves as part of this custom interface, guiding the user through the available methods and software for accessing and executing the Cement Microstructure Modelling and Analysis Package (CMMAP). As such, this manual provides a system overview, descriptions of the models present on the system, a description of the user interface program, and sample executions and graphical output. The user interface program has been developed so that the user may request a series of operations to be performed which will be stored in a script file that can later be executed using UNIX's script file facilities.

\section{SYSTEM OVERVIEW}

The computer imaging workstation consists of a SUN $3 / 160^{*}$ host minicomputer connected to a PIXAR II imaging computer. Instructions for start up of the system can be found in Appendix A. The PIXAR consists of its own processing unit and memory and a high resolution (1280*1024) monitor for displaying images of the models during and following execution. The SUN contains two hard disks, total capacity of 283 megabytes, and a 1/4"

- Certain commercial equipment is identified in this paper in order to adequately specify scientific procedures. In no case does such identification imply recommendation or endorsement by the National Institute of Standards and Technology, nor does it imply that the equipment is necessarily the best available for the purpose. 
tapedrive. The tapedrive is used for loading images from other systems such as the Building Materials Division's scanning electron microscope. The SUN operates under a Berkeley UNIX operating system environment $[1,2]$. Although the general user of the models will need only a passing acquaintance with UNIX, a summary of some of the more useful commands is provided in Appendix B of this manual.

The programs for the models are written in either $\mathrm{C}$ or Chap $\mathrm{C}$, an extension of standard $\mathrm{C}$ provided by PIXAR. The Chap $\mathrm{C}$ code is directly executable on the PIXAR's parallel processing unit. At runtime, the executable code is loaded from the SUN to the PIXAR and executed until completion. A brief description of the PIXAR capabilities follows; the interested reader should refer to PIXAR's user manuals [3] for more details and Appendix C for a listing of frequently used operating system-level commands provided by PIXAR. The PIXAR contains a large memory divided into discrete elements called pixels which are organized as a twodimensional array. The array contains $1280 * 1632$ elements of which $1280 * 1024$ are displayable on the PIXAR monitor at any one time. A layout of the PIXAR monitor and its coordinate system are provided in Fig. 1. The basic pixel consists of 4 separate channels of information: (red, green, blue, alpha or overlay). The alpha channel is used when overlaying one or more images to specify the relative transparency of each. Each channel may be assigned an integer value with values of 0 to 2048 being directly displayable as an intensity on the PIXAR monitor. For instance, bright red would be represented by the color quadruple $(2048,0,0,2048)$ whereas middle grey would be $(1024,1024,1024,2048)$.

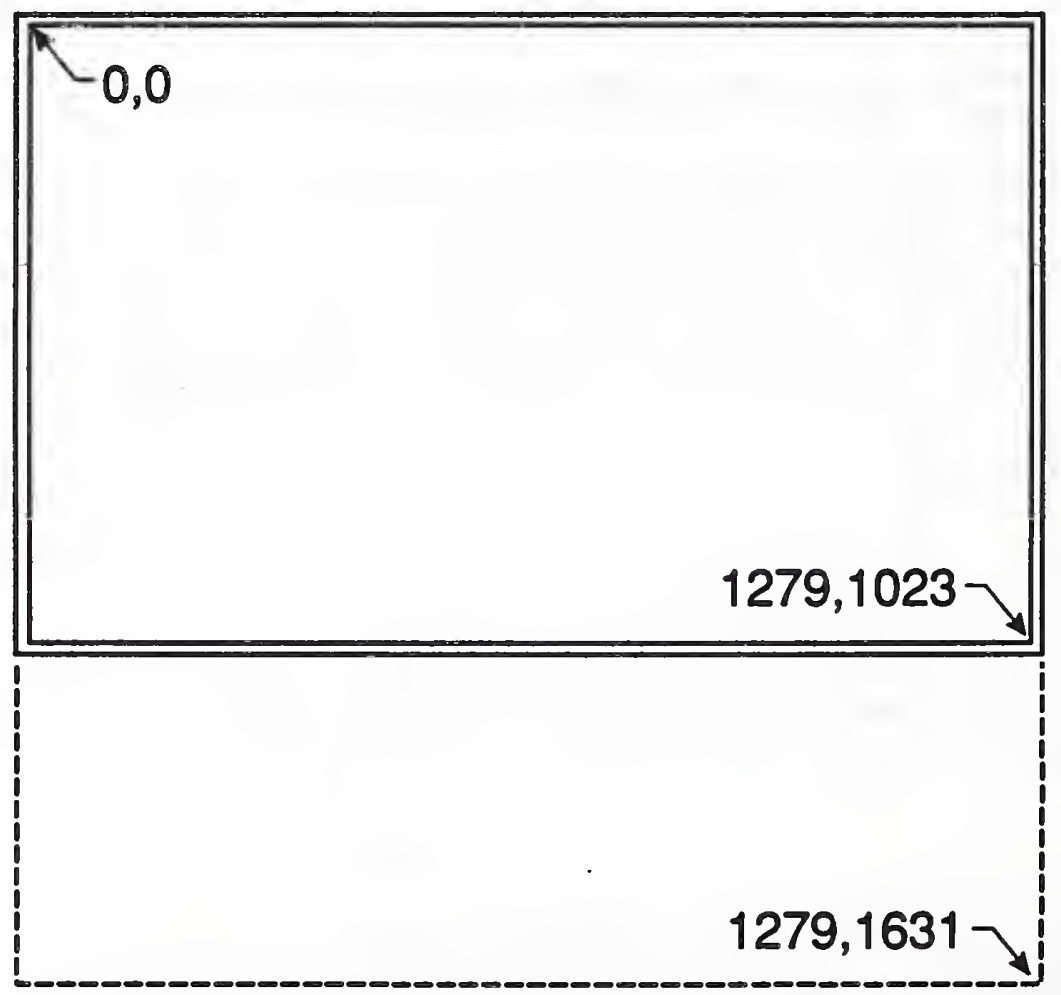

Figure 1 - Graphical View of Coordinate System of PIXAR Screen 
For modelling, this large memory can be used to store a representation of a cement microstructure or other systems of interest. Each pixel can represent an element of volume characterized by one set of attributes (i.e., a pixel may be assigned to pore space or hydration product but not to both). Movement can be simulated by giving a pixel the attributes of one of its neighbors. Thus, to simulate random walk diffusion, a diffusing species located at a given pixel is allowed to move to a neighboring pixel chosen at random. Each pixel can be accessed in a Chap $C$ program by a simple call to one of the routines in the Chap $C$ system library. Separate routines exist to read pixel values into the program and to write a new value from the program to a specific pixel. Using these routines, the system can be programmed to modify or operate on the representation of microstructure stored in the PIXAR memory. This allows processes such as hydration and mercury intrusion to be simulated and measurements such as connectivity and diffusivity to be performed.

For specific details on the various models, the reader is referred to the next section of this manual and the references provided in the bibliography in Appendix D.

\section{MODELLING AND ANALYSIS PROGRAMS}

In general, the microstructural models have been developed to execute in either two or three dimensions. For three dimensions, volume is represented as 100 two-dimensional slices each of which measures $100 * 100$ pixels (for a total of $1,000,000$ pixels or a $1000 * 1000$ matrix on the screen) as shown in Fig. 2, which depicts a starting three-dimensional microstructure consisting of spheres of a fixed size distribution. For two dimensions, any size area (within memory limits) can be utilized as the starting microstructure. Both the two-dimensional and three-dimensional versions of the models will be discussed below.

When the user begins the CMMAP program, the following main menu is presented:

0) Exit

1) Generate a starting microstructure

2) Run a hydration model

3) Compute diffusivities

4) Perform simulated mercury intrusion

5) Compute connectivity of pore space

6) Perform general microstructural characterization

7) Perform utility functions

Each of the above menu selections are discussed in detail in the sections which follow.

\subsection{Generate a Starting Microstructure}

There are seven options for creating a starting microstructure for the CMMAP system. In the program which provides the user interface, CMMAP.C, they are presented in the 


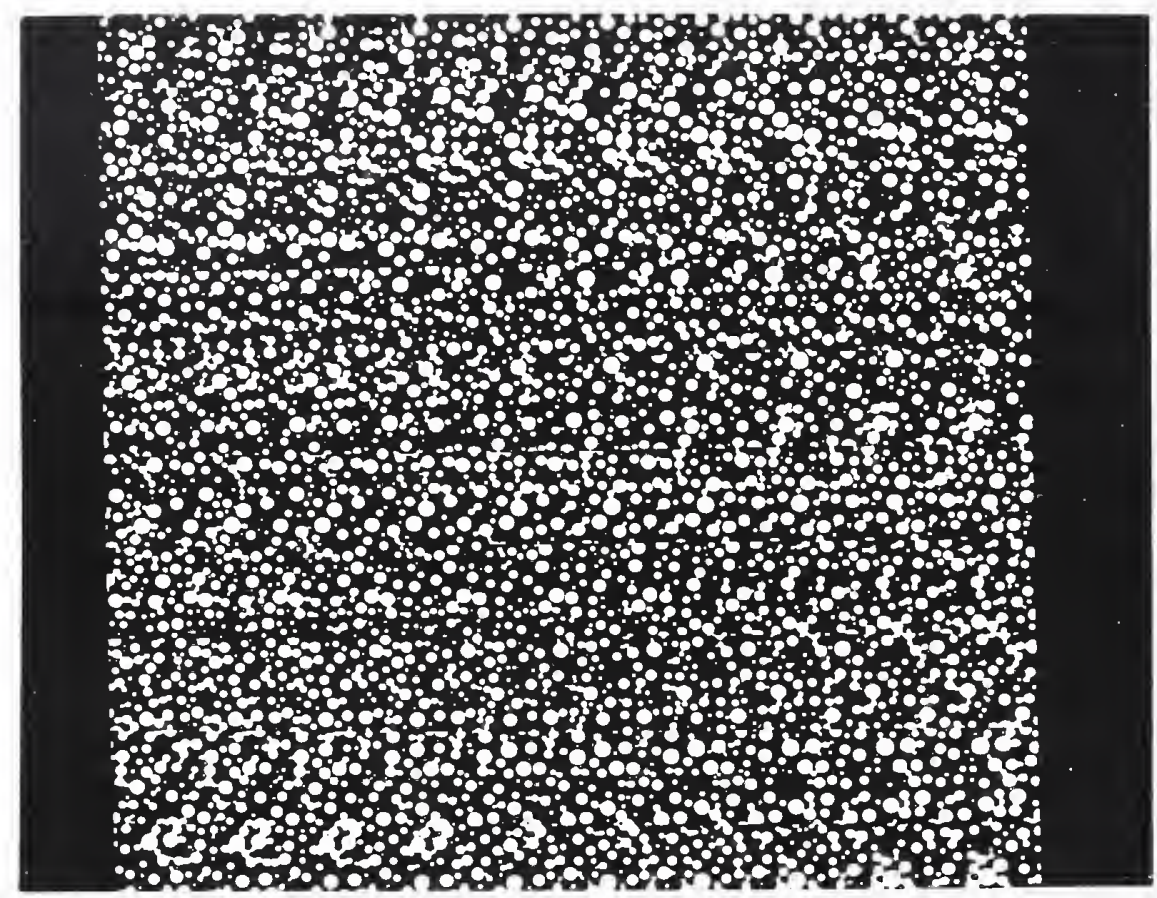

Figure 2 - Starting 3-D Microstructure Consisting of Randomly Parked Spheres Shown as 100 Slices, Each $100 * 100$ Pixels

following menu:

0) Exit to main menu

1) Create a 2-D microstructure using circles of discrete sizes

2) Create a 2-D microstructure using circles of a specified size distribution

3) Create a 2-D microstructure using cement particle shapes stored in a database

4) Retrieve a 2-D microstructure from disk

5) Create a 3-D microstructure using spheres of discrete sizes

6) Create a 3-D microstructure using spheres of a specified size distribution

7) Retrieve a 3-D microstructure from disk.

To execute option one, the user must supply the number of circles to be generated, their diameter, a randomization factor (since the circles are placed at random locations), and the coordinates (xlo xhi ylo yhi) of the rectangular area to place the circles within. At execution time, the circles will be randomly placed in the area such that they do not overlap. Periodic boundaries will be employed so that a circle that overlaps one edge of the area will be completed on the other side. The circles will be placed as red circles (pixel value $=[2048,0,0,2048]$ ) on a black background (pixel value $=[0,0,0,2048]$ ). 
If option two is selected, the user must supply the number of circles, the area coordinates, and the parameters which characterize the circle size distribution. The Weibull distribution is used for the size distribution so the user must supply three distributional parameters and a minimum and maximum radius to be generated. The actual equations utilized are provided in Appendix $\mathrm{E}$ which lists equations relevant to the models.

For option three, the user need only supply the number of particles to be placed and the area coordinates within which to place them. Actual SEM images of cement particles dispersed in an epoxy matrix have been stored in a datafile. This datafile is accessed at execution time to supply the requested number of particles to be randomly positioned. For option four, the user must supply the name of the image to be retrieved. This might be an image of a starting microstructure which the user has saved to disk, or an image saved by another user of the system. If the image has not been created previously on the PIXAR, the PIXAR software provides a conversion facility through the use of the see command outlined in Appendix C.

Since the volume size $(100 * 100 * 100)$ is fixed for the three-dimensional models, for option five, the user need only specify the number of spheres to be generated, their radius, and the maximum number of random attempts to be used in placing each sphere before an exhaustive search is conducted to find a suitable location for the sphere. Periodic boundaries are again employed during the sphere placement. Similar to the two-dimensional case, for option six, the user must specify the number of spheres and all parameters necessary to characterize the size distribution of spheres. To execute option seven, the user only supplies the name of the image to be retrieved from disk.

\subsection{Run a Hydration Model}

Once the starting microstructure has been established, the user may elect to execute the cement hydration model in either two or three dimensions. The model has been developed based on a few simple rules. Cement hydration is viewed as a three step process in which material dissolves off the surface of the starting particles, diffuses around in the pore network, and either nucleates or precipitates on a solid surface which may be part of the original starting particles or of some hydration product.

Both calcium hydroxide $\left(\mathrm{CH}^{*}\right)$ and calcium silicate hydrate $(\mathrm{CSH})$ are produced from the starting particles which are assumed to represent tricalcium silicate $\left(\mathrm{C}_{3} S\right)$. The calcium hydroxide is produced by a nucleation and growth process while the calcium silicate hydrate precipitates on $\mathrm{C}_{3} \mathrm{~S}$ and $\mathrm{CSH}$ solid surfaces. A hydration cycle consists of a dissolution followed by diffusion until all dissolved species have reacted to form hydration products. At the end of each cycle, the hydration program outputs the number of pixels present for each phase as well as a measure of the surface area (obtained by counting edges in two dimensions or faces in three

- Standard cement chemistry notation is used throughout this document : $\mathrm{C}=\mathrm{CaO}$ $\mathrm{H}=\mathrm{H}_{2} \mathrm{O} \quad \mathrm{S}=\mathrm{SiO}_{2}$ 
dimensions).

When the user elects to perform a hydration run, the following menu is presented:

0) Exit to main menu

1) Execute the 2-D hydration model

2) Execute the 3-D hydration model.

The user should be sure that they elect to perform the type of hydration (2-D or 3-D) corresponding to the type of microstructure they have created. If the user elects to execute a 2-D hydration they must supply the following parameters for the hydration program:

1) Method of performing volume expansion for $\mathrm{CSH}$ (since the CSH product occupies a larger volume than the starting $\mathrm{C}_{3} \mathrm{~S}$ reactant)

1- generating extra diffusing species during each dissolution

2- probabilistic-based expansion upon precipitation (reaction)

2) Boundary conditions

1- periodic boundaries at all edges

2- reflecting walls at all edges

3) Maximum probability (0.0-1.0) for $\mathrm{CH}$ nucleation (See Appendix E)

4) Exponential scale factor for $\mathrm{CH}$ nucleation (Appendix E)

5) Probability of diffusing $\mathrm{CSH}$ species to precipitate on a $\mathrm{C}_{3} \mathrm{~S}$ surface it contacts $(0.0-1.0)$

6) Probability of diffusing CSH species to precipitate on a CSH solid surface it contacts (0.0-1.0)

7) Number of cycles of hydration to perform

8) Maximum number of diffusion steps to be executed each cycle; when this number of diffusion steps has been exceeded, all remaining diffusing species nucleate in place into solid hydration products $(\mathrm{CH}$ or $\mathrm{CSH})$

9) Coordinates (xlo xhi ylo yhi) for hydration window (i.e., the window containing the starting microstructure)

10) Coordinates for each of two temporary storage windows to hold diffusing species' locations in the microstructure; these two windows should be the same size and should not overlap each other or the specified hydration window. The windows must be large enough to hold the locations of all diffusing species produced during a given cycle of the hydration. Thus, if these windows occupy the same amount of area as the hydration window, their size will be adequate. 
The user will also be prompted as to whether they want to send the output from the model execution to a file on disk as opposed to the CRT screen on the SUN workstation.

Since the 3-D hydration model is less flexible than the 2-D version, the user needs to specify less information:

1) Number of cycles of hydration to perform

2) Maximum number of diffusion steps to be executed each cycle

3) Maximum probability for $\mathrm{CH}$ nucleation

4) Exponential scale factor for $\mathrm{CH}$ nucleation.

Once again, if the user desires, they may have the output sent to a file instead of the CRT screen.

\subsection{Compute Diffusivities}

One key property of cement-based materials such as concrete is the diffusivity or diffusion coefficient. This property will influence such performance measures as resistance to sulfate attack and corrosion of steel reinforcement due to chloride ion penetration [4]. CMMAP provides the capability to compute the diffusivity of both 2-D and 3-D simulated microstructures. Specifically, the diffusivity of the capillary pore structure of the microstructure is computed. In real cement paste, diffusion may also occur through the gel pores of the CSH phase, but this contribution is not included in the programs on the PIXAR. However, algorithms exist on other computers at NIST to include the CSH phase in the diffusivity computation.

When the user selects this option from the main menu, the following options are presented:

0) Exit to main menu

1) Compute 2-D diffusivity using blind ant algorithm

2) Compute 2-D diffusivity using y-del algorithm

3) Compute 3-D diffusivity using blind ant algorithm.

Before detailing the parameters required for each of these options, we shall first provide an overview of the two methods employed for estimating diffusivity, the blind ant and the y-del algorithms.

In the blind ant method, random walkers, called "blind" ants, are released and allowed to wander throughout the pore network of a microstructure [5]. This method is implemented by randomly locating a large number of ants at pore space pixels. At each time step, each ant attempts to randomly move to one of its neighboring locations, where there are four neighbors 
in 2-D and six in 3-D. If the new location is also pore space, the move is allowed, but if the new location is solid material, the ant remains at its original location (hence the term blind ants since they cannot see where they are going). After a large number of steps, the program determines the average distance squared that each ant has progressed from its starting location. This value is a linear function of the number of steps with a slope proportional to the diffusion coefficient, D. Multiplying D by the effective porosity of the microstructure provides the effective diffusivity. The disadvantage of this method in comparison to other computer methods is that a large number of steps (on the order 100,000 ) are required to accurately estimate the diffusivity of a complex pore system. The method is equally applicable to 2-D and 3-D microstructures.

The y-del algorithm considers the pore network of the microstructure as an electrical network where neighboring pore pixels are connected by a conductor. Using the y-del resistor transformation, this complex network of bonds is reduced to a single bond of conductance equal to the relative diffusivity of the original microstructure $[6,7]$. The relative diffusivity is defined as the ratio of the measured diffusivity to that obtained for a porosity of $100 \%$. Since bonds may exist in any direction, the memory overhead of this method is quite large. Thus, currently this method can be executed accurately only on two-dimensional microstructures of size less than $200 * 200$ pixels on the SUN/PIXAR system. However, three-dimensional algorithms do exist and are available on other computer systems at the NIST site for those interested in quickly estimating the diffusivity of 3-D and larger 2-D microstructures.

If the user elects to estimate diffusivity in 2-D using the blind ant algorithm, they must specify the number of blind ants to use, the number of steps for each ant to take, whether to use periodic boundaries or reflecting walls, and the coordinates of the image in which to run the ants. In 3-D, the user need only specify the first two of the above listed parameters as the image size is fixed at $100 * 100 * 100$ and periodic boundaries are automatically employed. For the 2-D y-del algorithm, the user needs to supply the coordinates of the original image window and coordinates for windows to hold both vertical and horizontal bond information from the original microstructure. The two bond windows must be slightly larger (at least +3 in both directions) than the original microstructure window to allow for the placement of "electrodes" at the edges. To properly execute the $y$-del code, the original microstructure image must consist of a black pore network (pixel value $=[0,0,0,2048]$ ) with any non-black background.

\subsection{Perform Simulated Mercury Intrusion}

Mercury porosimetry is a widely used technique for characterizing the pore structure of cement-based and other porous materials [8]. By increasing the external pressure, the mercury is able to flow through smaller and smaller pore necks and fill a larger pore volume in the sample, as the mercury meniscus diameter is inversely proportional to external pressure. Typical output consists of a volume intruded vs. pore diameter curve as shown in Fig. 3.

Given a representation of pore structure stored in the computer, mercury intrusion can be simulated by directly varying the meniscus diameter. In $2-\mathrm{D}$, this simulation is implemented 


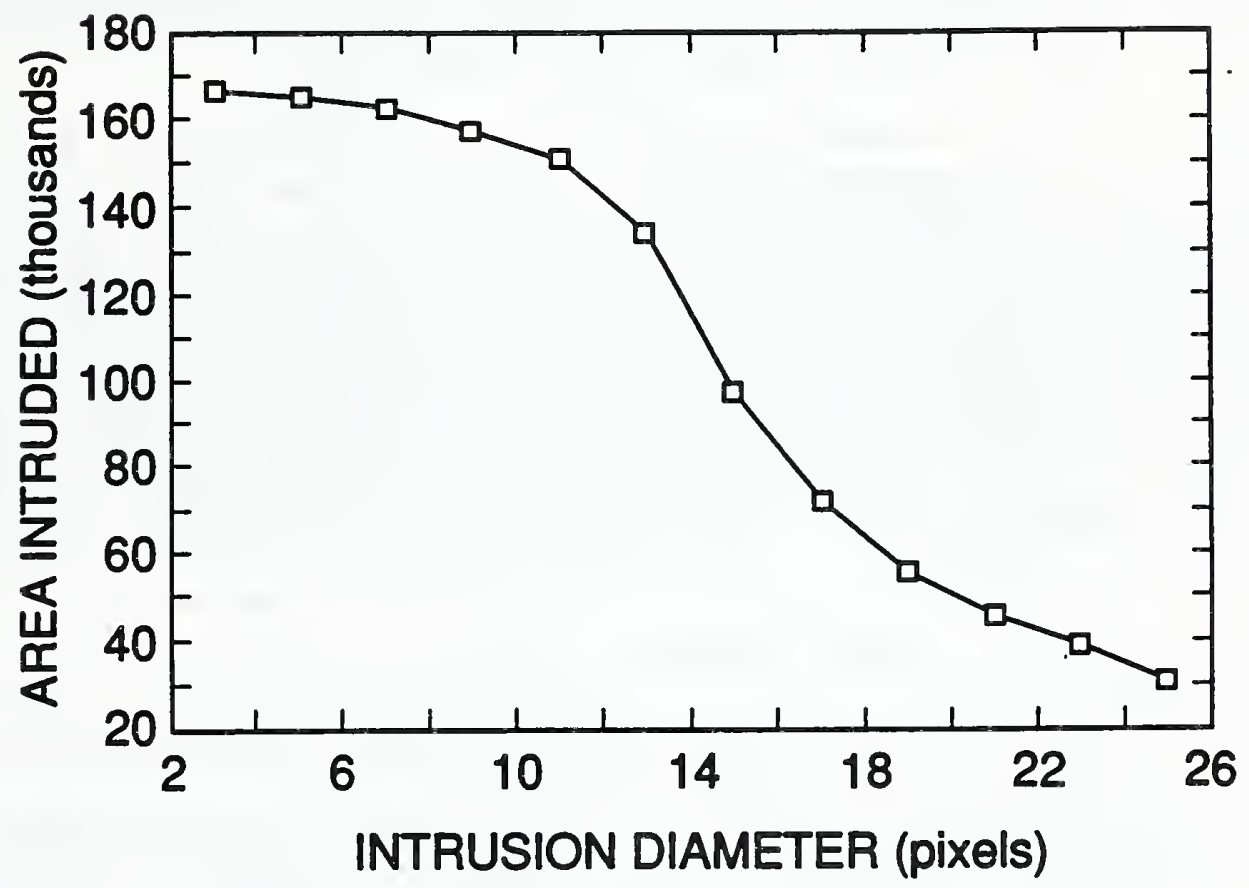

Figure 3 - Two-dimensional Mercury Intrusion Curve Produced by CMMAP

as follows. First, the original microstructure image containing black pores and red solid material is copied to a new location on the PIXAR screen. When necessary, to obtain such an image, the program usr/pixar/sem/thrall3 described in the specialty PIXAR programs section of this manual can be used. This new working image is surrounded by a bath of "active" mercury which is green $(0,2048,0,2048)$ in color. A diameter for the meniscus is selected and the mercury is allowed to penetrate into the pore structure until further flow is restricted. Basically, this is executed in an iterative process by propagating the flow from all active mercury (green) pixels. For each active mercury pixel, a circle of the meniscus diameter is overlaid on the image centered at the active mercury pixel location. If the circle is entirely contained in pore space, the mercury can propagate ahead. This propagation is executed by turning all pore pixels within the circle green (so that they will be active mercury for the next iteration) and turning the starting active mercury pixel yellow $(2048,2048,0,2048)$ to indicate inactive mercury which does not need to be checked for further propagation.

When only yellow pixels are present in the pore structure, no further flow is possible and the area intruded can be determined. This algorithm can then be repeated on a new copy of the working image with a larger meniscus diameter until the complete mercury intrusion curve is obtained. An additional parameter of interest is the meniscus diameter at which the mercury can no longer flow from one side of the microstructure image to the other, representing a critical 
pore diameter for flow in the microstructure. This can be determined by executing the above algorithm, but initially surrounding only one side of the microstructure with mercury as opposed to all four sides, and determining if the mercury is able to flow to the opposite side by checking for the presence of yellow (inactive mercury) pixels at that edge.

To execute a mercury intrusion simulation in 2-D, the user must specify coordinates for four image windows: two temporary storage windows, the original image, and the working image. The temporary storage windows are used to store the locations of all pixels which are part of the propagating mercury front (i.e. green pixels). In addition, the user needs to supply the range of meniscus radii over which to execute the intrusion process by giving the minimum and maximum values. For the intrusion simulation, the meniscus diameter is determined as two times the radius + one. This equation is used so that each "intruding circle" can be centered on a pixel. As with many of the other models, the user has the option of sending the output of the mercury intrusion simulation to either the CRT screen or to a file on disk for more permanent storage.

At this time, a 3-D simulation of mercury intrusion is not available. In addition to the large memory overhead required, there is also a theoretical difficulty. There is only one radius of curvature for meniscii in two dimensions, which uniquely defines a circle. In three dimensions, two radii of curvature are required to define a meniscus, so that ellipsoidal shapes are possible in addition to spherical shapes. Thus, simply repeating the two-dimensional algorithm with spheres is not completely accurate. Hopefully, this problem will be remedied in the near future and a 3-D mercury intrusion simulation will be added to the CMMAP package. Currently, a 3-D spherical intrusion package, which is only an approximation to actual mercury intrusion, is available on other computers, such as the Cyber supercomputer, at the NIST site.

\subsection{Compute Connectivity of Pore Space}

The pore network in cement and concrete influences most of these materials' ultimate properties including compressive strength, permeability, and resistance to chemical attack [9-11]. As the hydration process proceeds, the pore system evolves from a very open system of connected pores to a capillary network system of pores which may or may not be interconnected. Assessing the connectivity of the pore space is thus one method for characterizing a computer simulated microstructure. There are three options for analyzing connectivity presented by program CMMAP:

0) Exit to main menu

1) Compute 2-D connectivity of pore space in 2-D microstructure

2) Compute 2-D connectivity of pore space in 3-D microstructure

3) Compute 3-D connectivity of pore space in 3-D microstructure.

To assess connectivity, the microstructure must contain black (value $=[0,0,0,2048]$ ) pores. The connectivity of other phases ( $\mathrm{CSH}, \mathrm{CH}$, etc.) can also be assessed by thresholding a microstructure to highlight the phase of interest in black, with the remainder of the 
microstructure some other color (such as red). The program usr/pixar/sem/thrall3 described in Section 3.7.1 can be utilized to perform the threshold operation. The connectivity is measured by determining what fraction of the pore space is part of a continuous pathway from the top to the bottom of the microstructure via a "burning" algorithm [12]. Both the amount of pore space accessible from the top and the amount of pore space "connected" from top to bottom are computed by the analysis programs.

To execute option 1, the user must specify the coordinates of the microstructure representation and coordinates for two identically sized temporary storage windows. The temporary storage windows are used to store the locations of all pixels which are part of the current "burning front". The program first turns all non-black space to red and then executes the burning algorithm to assess connectivity. Thus, if the original image is going to be needed later, the user should save it to disk before executing this analysis. As described earlier, the user also has the option of sending the analysis output to a file on disk. For options 2 and 3, the user need only specify if they wish to send the output to disk. To determine 2-D connectivity of a 3-D microstructure, the pore connectivity of each of the 100 slices is considered separately and results for all slices are output to the device of the user's choice.

\subsection{Perform General Microstructural Characterization}

The microstructural characterization submenu presents two options for characterizing 2-D microstructures or 2-D slices of 3-D microstructures. The user may choose to:

0) Exit to main menu

1) Characterize discrete objects by size, location, etc.

2) Characterize complex objects by autocorrelation analysis.

Option 1 basically provides a size distribution for discrete phases such as $\mathrm{C}_{3} \mathrm{~S}$ or $\mathrm{CH}$ in a 2-D microstructure. For more complex (continuous) phases, autocorrelation analysis provides one method for characterization.

Autocorrelation analysis examines the spatial arrangement of a complex phase by determining the correlation of a phase with itself as shown by the equations provided in Appendix E. This is accomplished by making a copy of the original image, shifting it various amounts in the $\mathrm{x}$ and $\mathrm{y}$ directions, overlaying it on the original image, and measuring the amount of phase overlay in the overlaid image. With no shift, this value will be simply the phase fraction in the original image. As the shift value increases, eventually, any correlation will be due only to randomness and the measured phase fraction will approach a value of the original phase fraction squared, the value to be expected if two totally uncorrelated images are overlaid. The manner in which this asymptotic value is approached provides information on a variety of parameters such as specific surface, fractal dimension, etc. $[13,14]$.

To execute size distribution analysis, the user must specify the coordinates of the image to be analyzed and coordinates for two temporary storage windows. Additionally, the analysis 
can be sent either to the CRT screen or to a file on disk. For autocorrelation analysis, the user must provide coordinates for the original image and an area to hold the shifted image, a filename to hold the intermediate results, and the maximum shift to be used in calculating the autocorrelation. This intermediate file can then be analyzed to determine the actual autocorrelation curve by running the $\mathrm{C}$ program autocalc by simply typing autocalc at the UNIX prompt.

\subsection{Perform Utility Functions}

The main menu of CMMAP also provides the user the option of executing utility functions such as image manipulation, execution of general purpose programs, and execution of UNIX commands. The submenu for this function consists of:

0) Exit to main menu

1) Clear the PIXAR screen

2) Copy a portion of the PIXAR screen to a new location

3) Save an image to disk

4) Retrieve an image from disk

5) Remove an image from disk

6) Execute a CHAP C program

7) Execute a UNIX operating system level command

When the above options are selected, the user is prompted to enter either coordinates, filenames, or complete commands as required by the specific function selected. A brief description of some of the specialty programs for the PIXAR follows.

\subsubsection{Specialty PIXAR Programs}

/usr/pixar/sem/thrall3- thresholds an image to produce red objects on a black background (or vice versa depending on your viewpoint). The user specifies the coordinates of the image to be thresholded and a set of color limits (one set of low and high values to be used for all three of the red, green, and blue channels) to use in specifying the threshold criteria. Pixels whose color value is contained in the threshold range are made red while all others are made black.

/usr/pixar/cmmap/countblack- determines the percentage of pixels in a user-specified window which are black. For example, porosity can be quickly assessed by simply providing this program with the coordinates of the image window to be analyzed.

/usr/pixar/cmmap/countred- similar to countblack, except it counts red pixels in the user-specified image window.

/ust/pixar/cmmap/cbtpixar- general-purpose imaging program for manipulating a source image located within coordinates $0,511,0,511$. 
/usr/pixar/cmmap/colortwo- program used to draw an inert square aggregate (aqua in color) of user-specified size in the middle of the window specified by coordinates $200,700,200,700$.

/usr/pixar/cmmap/porfluct- program to determine various phase fractions as a function of distance from aggregate surface for analyzing hydration in the cement paste-aggregate interfacial zone.

\section{USER INTERFACE}

The user interface for CMMAP is provided by the program CMMAP.C which can be accessed directly from the SUN/PIXAR system. Basically, the user utilizes CMMAP.C to create a script file, which can be executed whenever the necessary SUN/PIXAR resources are available. After execution, the output files created are available to the user for their evaluation. To access CMMAP.C, the user simply types CMMAP at the UNIX prompt.

\section{EXAMPLE SESSIONS AND OUTPUT}

The capabilities and operation of CMMAP will be demonstrated through a series of three examples. Transcripts of the actual CMMAP sessions to create these examples can be found in Appendix F.

Session 1

For the first example, a 2-D microstructure consisting of 240 circles each of a diameter of 21 pixels is generated, 2-D connectivity is assessed, and mercury intrusion is simulated for meniscus radii ranging from 1 to 12 (or diameters of 3 to 25) pixels. The actual script file, exam001.scr, created by CMMAP to perform these operations is as follows:

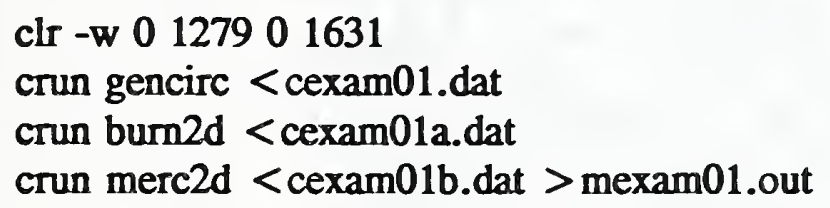

From a notation standpoint, $<$ and $>$ indicate redirection of the standard input and standard output devices respectively. The first instruction in this script file simply clears the entire PIXAR screen. This is always a good idea when starting a new simulation run as the user cannot be sure of the state of the PIXAR screen.

The second instruction creates a starting 2-D microstructure based on the data in the file cexam01.dat, whose layout is:

$\begin{array}{ll}240 & \text { (number of circles to place) } \\ 21 & \text { (diameter of circles in pixels) } \\ 0 & \text { (number of random numbers to generate at start) } \\ 05000500 & \text { (coordinates of box in which to place circles) }\end{array}$


Based on this data, the original microstructure shown in Fig. 4 is generated.

The third instruction in the script file executes a 2-D connectivity analysis of the pore structure shown in Fig. 4, based on the data in the file cexam01a.dat, whose layout is:

05000500

60010990499

6001099500999 (coordinates of image to analyze)

(coordinates of temporary storage window \#1)

(coordinates of temporary storage window \#2)

When this command is executed, the following results are returned to the console:

Top- $167178 \quad$ Through- 167178.

Thus, for this starting microstructure, 167178 out of 167241 total (as measured by separate execution of the program countblack) pore pixels are part of a connected pathway from the top to the bottom of the image. This quantitative analysis agrees with one's qualitative assessment of the openness of the pore structure shown in Fig. 4.

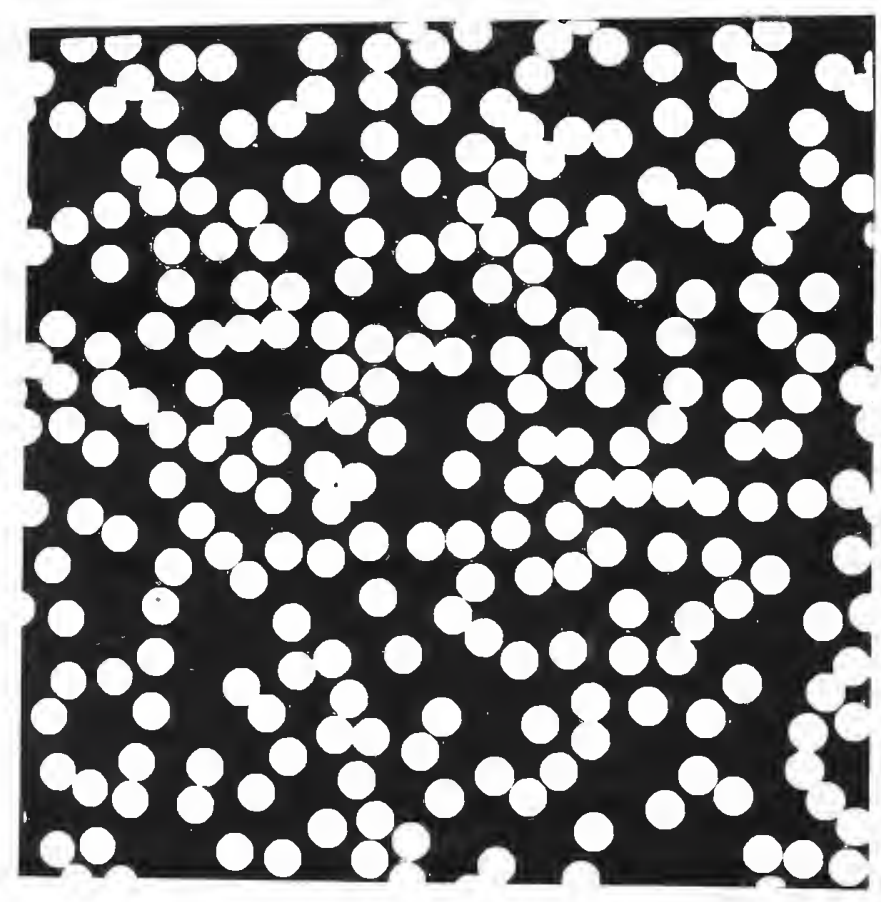

Figure 4 - Starting 2-D Microstructure Consisting of Randomly Parked Monosize Circles

The final instruction in the file exam001.scr executes a mercury intrusion simulation on the starting microstructure, based on data in the file cexam01b.dat, whose layout is as follows:

0499600799

(coordinates of temporary storage window \#1) 
0499800999

05000500

6001100100600

112 (coordinates of temporary storage window \#2)

(coordinates of original image to be intruded)

(coordinates of working image to be intruded)

(minimum and maximum meniscus radii for intrusion simulation)

The output from the mercury intrusion, shown in graphical form in Fig. 3, is sent to the file mexam01.out. After execution, this file contains the following information:

total for 1 is 166469

Connected top to bottom 1

Connected left to right 1

total for 2 is 164825

Connected top to bottom 1

Connected left to right 1

total for 3 is 162234

Connected top to bottom 1

Connected left to right 1

total for 4 is 156871

Connected top to bottom 1

Connected left to right 1

total for 5 is 150423

Connected top to bottom 1

Connected left to right 1

total for 6 is 133680

Connected top to bottom 1

Connected left to right 0

total for 7 is 96804

Connected top to bottom 0

Connected left to right 0

total for 8 is 71816

total for 9 is 55590

total for 10 is 45575

total for 11 is 39359

total for 12 is 30965 .

The "total" lines of data indicate the area intruded for a given meniscus radius (diameter $=2^{*}$ radius +1 ) while the "connected" lines of data indicate whether for a given meniscus radius, a connected pathway exists across the microstructure (either top to bottom or left to right). Here, a 1 indicates that a connected pathway does exist, while a 0 indicates that one does not. From the results, the critical threshold radius for this image is between 6 and 7 pixels, as it is at a meniscus radius of 7 pixels (diameter of 15 pixels) that for the first time no connected mercury pathways exist across the microstructure image. This critical diameter approximately corresponds to the inflection point of the mercury intrusion curve shown in Fig. 3. Figs. 5 and 6 provide graphical illustrations of the final intruded images for meniscus radii 


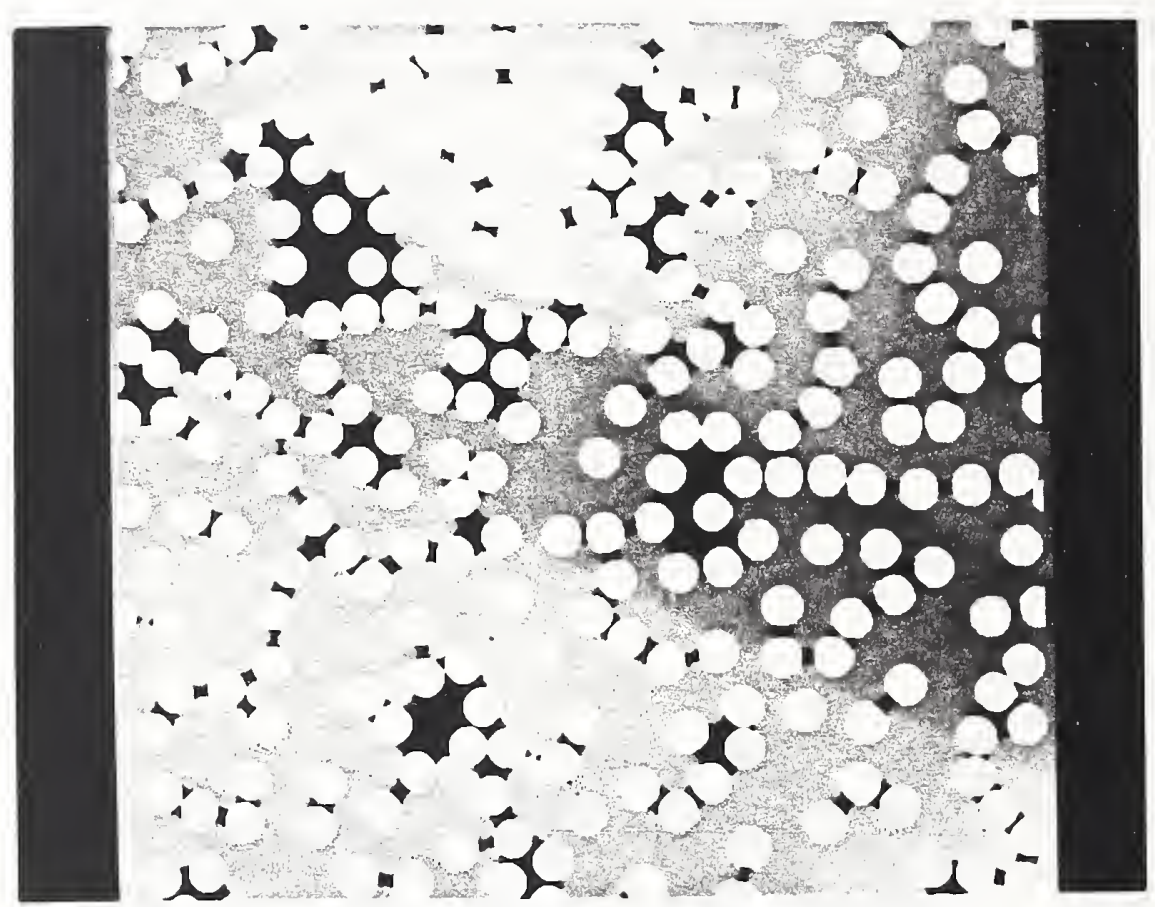

Figure 5 - Fig. 4 After Intrusion by Mercury with Meniscus Radius Set at Five Pixels or a Meniscus Diameter of Eleven Pixels

of 5 and 8 pixels respectively. In these figures, black indicates unintruded pore space while grey indicates mercury-filled porosity. These figures support the threshold radius mentioned earlier as the former contains a connected pathway of mercury across the image while the latter does not.

\section{Session 2}

For example 2, a 3-D microstructure consisting of spheres of a specified size distribution is generated, 3-D connectivity and diffusivity are assessed, 20 cycles of hydration are performed, and 3-D connectivity is reevaluated for the hydrated microstructure. The actual script file, exam002.scr, created by CMMAP to perform these operations is as follows:

clir -w 0127901631

crun gn3dsizd < cexam02a.dat

crun burn3d $>$ cexam02a.out

crun antdif3d < cexam02b.dat

crun cmhyd3d1 <cexam02c.dat > cexam02b.out

crun burn3d >cexam02c.out 


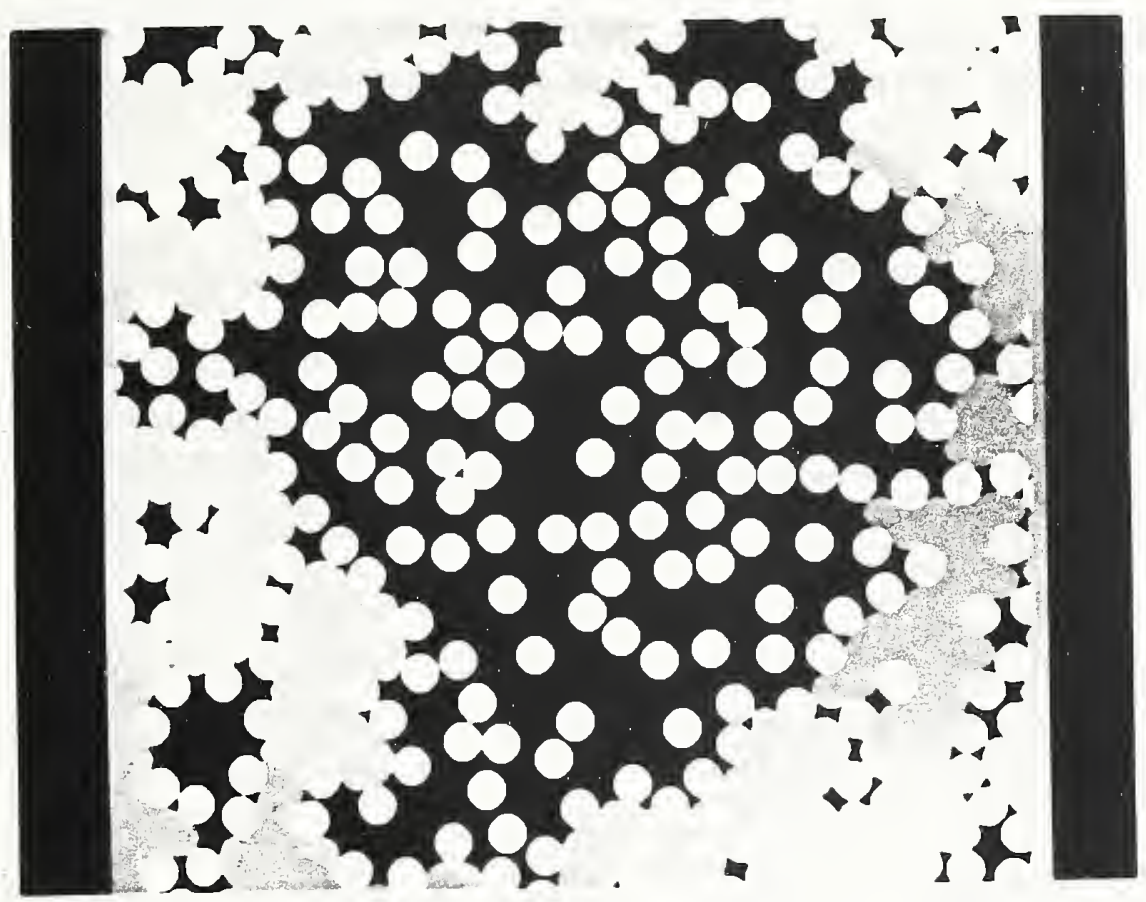

Figure 6 - Fig. 4 After Intrusion by Mercury with Meniscus Radius Set at Eight Pixels or a Meniscus Diameter of Seventeen Pixels

The first instruction is used to clear the PIXAR screen before beginning the simulation. The second instruction creates a 3-D starting microstructure based on the data in the file cexam02a.dat, whose layout is:

500

2.00

4.00

1.00

19

500 (number of spheres to be generated)

( $\alpha$ parameter for Weibull distribution - See Appendix E)

( $\beta$ parameter for Weibull distribution)

( $\gamma$ parameter for Weibull distribution)

(minimum and maximum radii of spheres to be created)

(maximum number of random attempts to be used in placing a sphere)

Based on this data, the original microstructure shown in Fig. 2 is created.

The third instruction in exam002.scr executes an assessment of the 3-D connectivity of the pore space in Fig. 2. The results are sent to a file designated as cexam02a.out and are as follows:

Top- 614627 Through- 614621 .

Thus, 614,621 pore pixels are part of the connected pathway through the 3-D microstructure 
from the top to the bottom.

The fourth instruction in this script file determines the diffusivity of the 3-D microstructure using the blind ant algorithm. The data file, cexam02b.dat, used for this analysis is as follows:
10000
(number of ants to use)
100000
(number of steps for each ant to take)

The results of this analysis are automatically written to a data file (after every 500 steps) named ant3d.dat. Results for this particular simulation are shown in Fig. 7 which provides a plot of the average distance squared travelled by each ant vs. the number of steps taken. From the slope of this line, the effective (relative) diffusivity can be determined to be 0.4467 .

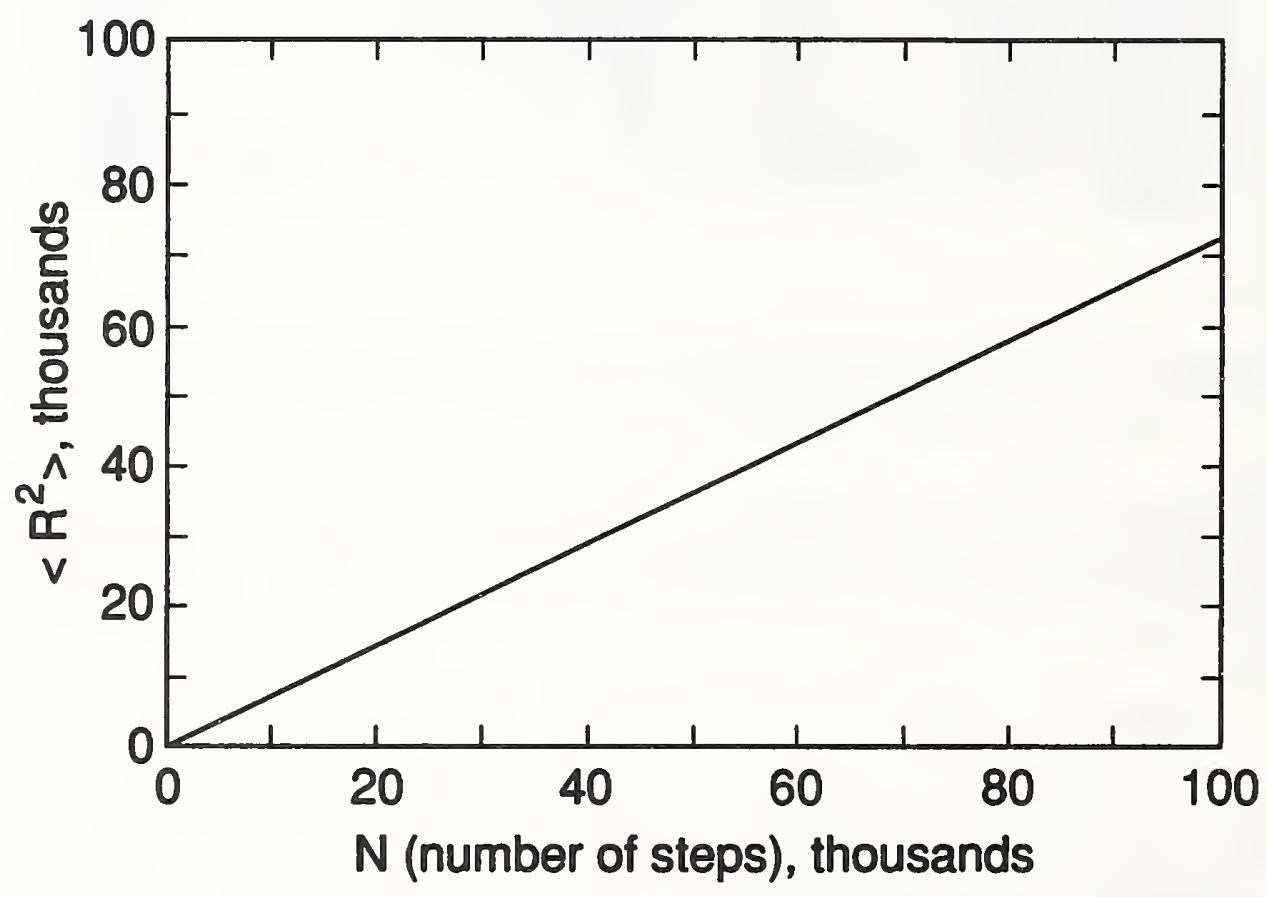

Figure 7 - Results of Blind Ant Diffusivity Analysis of the 3-D Microstructure Shown in Fig. 2

The fifth instruction in this script file executes a hydration of the 3-D microstructure, based on input data in the file cexam02c.dat, which is as follows:

20 (number of cycles of hydration to execute)

10000 (maximum number of random diffusion steps per cycle) 
0.001 (maximum probability for $\mathrm{CH}$ nucleation- See Appendix E)

100000. (exponential scale factor for $\mathrm{CH}$ nucleation)

The output created by the hydration program is sent to a file named cexam02b.out and is summarized in Table I.

Table I

Summary of 3-D Hydration Results

\begin{tabular}{||c|c|c|c|c|c|c||}
\hline Cycle & Dissolved & CH & CSH & Pore & C3S & Surface \\
\hline 1 & 116164 & 0 & 0 & 614724 & 385276 & 272134 \\
\hline 2 & 71664 & 30675 & 85489 & 548848 & 334988 & 631804 \\
\hline 3 & 57123 & 49599 & 138229 & 508208 & 303964 & 839936 \\
\hline 4 & 47792 & 64683 & 180268 & 475814 & 279235 & 990062 \\
\hline 5 & 42101 & 77303 & 215440 & 448712 & 258545 & 1102452 \\
\hline 6 & 37842 & 88420 & 246424 & 424837 & 240319 & 1192889 \\
\hline 7 & 33832 & 98413 & 274273 & 403377 & 223937 & 1267146 \\
\hline 8 & 29555 & 107347 & 299171 & 384191 & 209291 & 1327406 \\
\hline 9 & 26687 & 115151 & 320922 & 367431 & 196496 & 1376691 \\
\hline 10 & 23831 & 122198 & 340562 & 352297 & 184943 & 1418055 \\
\hline 11 & 21643 & 128491 & 358100 & 338783 & 174626 & 1453117 \\
\hline 12 & 19431 & 134206 & 374028 & 326510 & 165256 & 1483322 \\
\hline 13 & 17840 & 139337 & 388328 & 315491 & 156844 & 1509025 \\
\hline 14 & 16326 & 144048 & 401457 & 305374 & 149121 & 1531403 \\
\hline 15 & 14990 & 148359 & 413472 & 296116 & 142053 & 1550643 \\
\hline 16 & 13898 & 152317 & 424504 & 287616 & 135563 & 1567812 \\
\hline 17 & 12743 & 155987 & 434732 & 279735 & 129546 & 1582785 \\
\hline 18 & 11673 & 159352 & 444110 & 272509 & 124029 & 1596273 \\
\hline 19 & 10706 & 162423 & 452701 & 265890 & 118975 & 1608250 \\
\hline 20 & 9986 & 165261 & 460580 & 259819 & 114340 & 1618905 \\
\hline & $\cdots--$ & 167898 & 467929 & 254156 & 110017 & $\cdots--$ \\
\hline
\end{tabular}

The values for $\mathrm{CH}, \mathrm{CSH}$, pore, and $\mathrm{C}_{3} \mathrm{~S}$ indicate the number of pixels of each present at the beginning of the cycle. The values for surface indicate the number of surface area pixel faces present at the beginning of the cycle. The image of one of the 100 slices of the 3-D microstructure after 20 cycles of hydration is shown in Fig. 8.

The final instruction in the script file executes an assessment of the 3-D connectivity of 


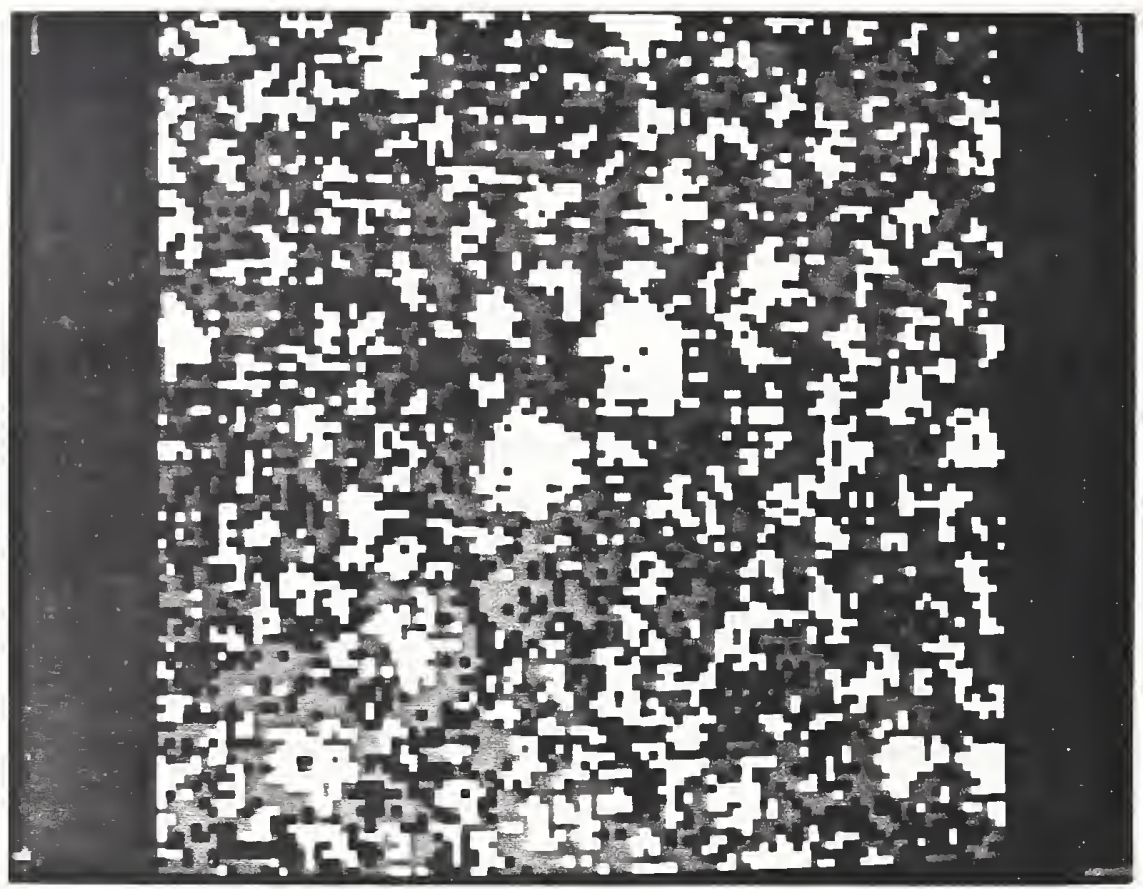

Figure 8 - Image of One of the 100 Slices of the 3-D Microstructure Shown in Fig. 2 After 20 Cycles of Hydration by CMMAP

the pore space for the hydrated microstructure. The results are sent to a file designated as cexam02c.out and are as follows:
Top- 193938
Through- 192111.

Thus, despite extensive hydration, the porosity remains partially connected in three dimensions, as 192,111 out of 254,156 or about $76 \%$ of the porosity is part of a connected pathway through the 3-D microstructure.

Session 3

For example 3, a 2-D microstructure consisting of actual cement particles is created, 2-D diffusivity is measured using the y-del algorithm, hydration is performed, diffusivity is reassessed, and the final porosity is characterized using autocorrelation analysis. The actual script file, exam003.scr, created by CMMAP to perform these operations is as follows:

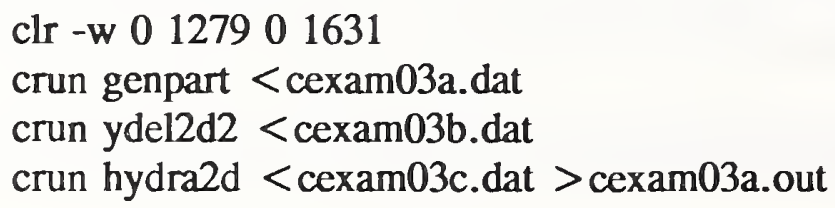


crun ydel2d2 < cexam03d.dat

crun /usr/pixar/sem/thrall3

crun /usr/pixar/sem/thrall3

crun autocem < cexam03e.dat

autocalc

After the first instruction clears the PIXAR screen, the second creates the starting 2-D microstructure based on the data in the file cexam03a.dat, whose layout is:

264

01500150

0

(number of particles to be generated)

(coordinates specifying box within which to place particles)

(number of random numbers to generate at start)

Based on this data, the original microstructure shown in Fig. 9 is created. This microstructure corresponds to a water-to-cement ratio of 0.5 .

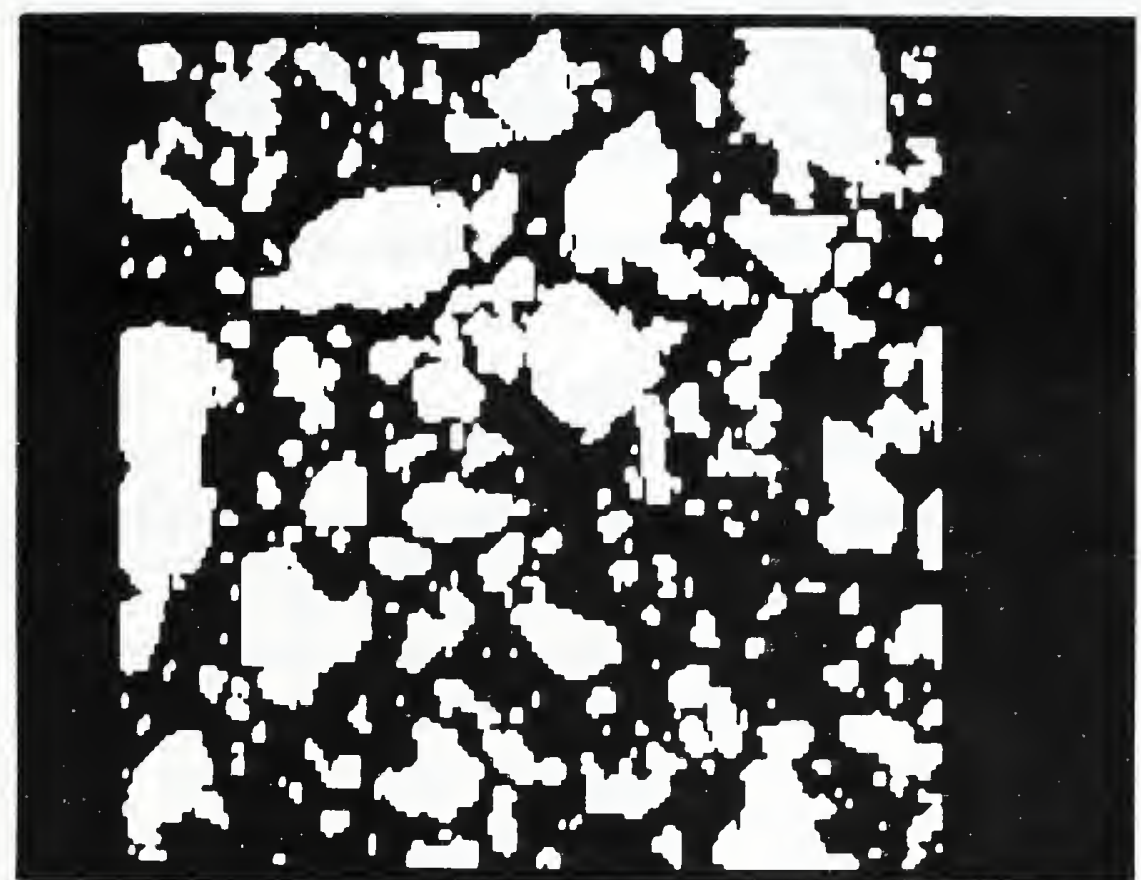

Figure 9 - Starting 2-D Microstructure Consisting of Randomly Parked Cement Particle Shapes

The third instruction determines the diffusivity for this 2-D microstructure using the $y$-del algorithm. The data file cexam03b.dat contains the parameters required to execute this analysis and is organized as follows: 
01500150

200360100260

200360300460 (coordinates of original image to be analyzed)

(coordinates of box to hold horizontal bonds)

(coordinates of box to hold vertical bonds)

When executed, this instruction returns a value of 0.169 for the diffusivity of this 2-D microstructure.

The fourth instruction in exam003.scr executes the 2-D hydration model, based on input data in the file cexam03c.dat, whose layout is:

1

2

0.005

50000 .

1.0

1.0

4

10000

01500150

5009990499

500999500999 (coordinates of diffusing species window \#2)
(ID of method for performing volumetric expansion)

(ID for periodic boundaries vs. reflecting walls)

(maximum probability for $\mathrm{CH}$ nucleation - See Appendix E)

(exponential scale factor for $\mathrm{CH}$ nucleation)

(probability of diffusing $\mathrm{CSH}$ sticking to solid $\mathrm{C}_{3} \mathrm{~S}$ )

(probability of diffusing CSH sticking to solid CSH)

(number of cycles of hydration to execute)

(maximum number of random diffusion steps per cycle)

(coordinates of window on which to perform hydration)

The output created by hydra2d is stored in the file cexam03a.out, and is summarized in Table II.

Table II

Summary of 2-D Hydration Results

\begin{tabular}{|c|c|c|c|c|c|c|}
\hline Cycle & Dissolved & CH & CSH & Pore & $\mathrm{C}_{3} \mathrm{~S}$ & Surface \\
\hline 0 & 0 & 0 & 0 & 14053 & 8748 & $-\cdots$ \\
\hline 1 & 3686 & 973 & 2713 & 11963 & 7152 & 5159 \\
\hline 2 & 1674 & 1415 & 3945 & 11014 & 6427 & 8717 \\
\hline 3 & 1376 & 1778 & 4958 & 10234 & 5831 & 10245 \\
\hline 4 & 1138 & 2078 & 5796 & 9589 & 5338 & 11171 \\
\hline
\end{tabular}

Here, surface values indicate the number of surface area pixel edges present at the beginning of each cycle. The image of the microstructure produced after 4 cycles of hydration is shown in Fig. 10. Like instruction 3, instruction 5 of exam003.scr assesses the diffusivity of the 2-D hydrated microstructure. Since the porosity has become disconnected in 2-D, a value of 0 is returned for the diffusivity when this instruction is executed. 


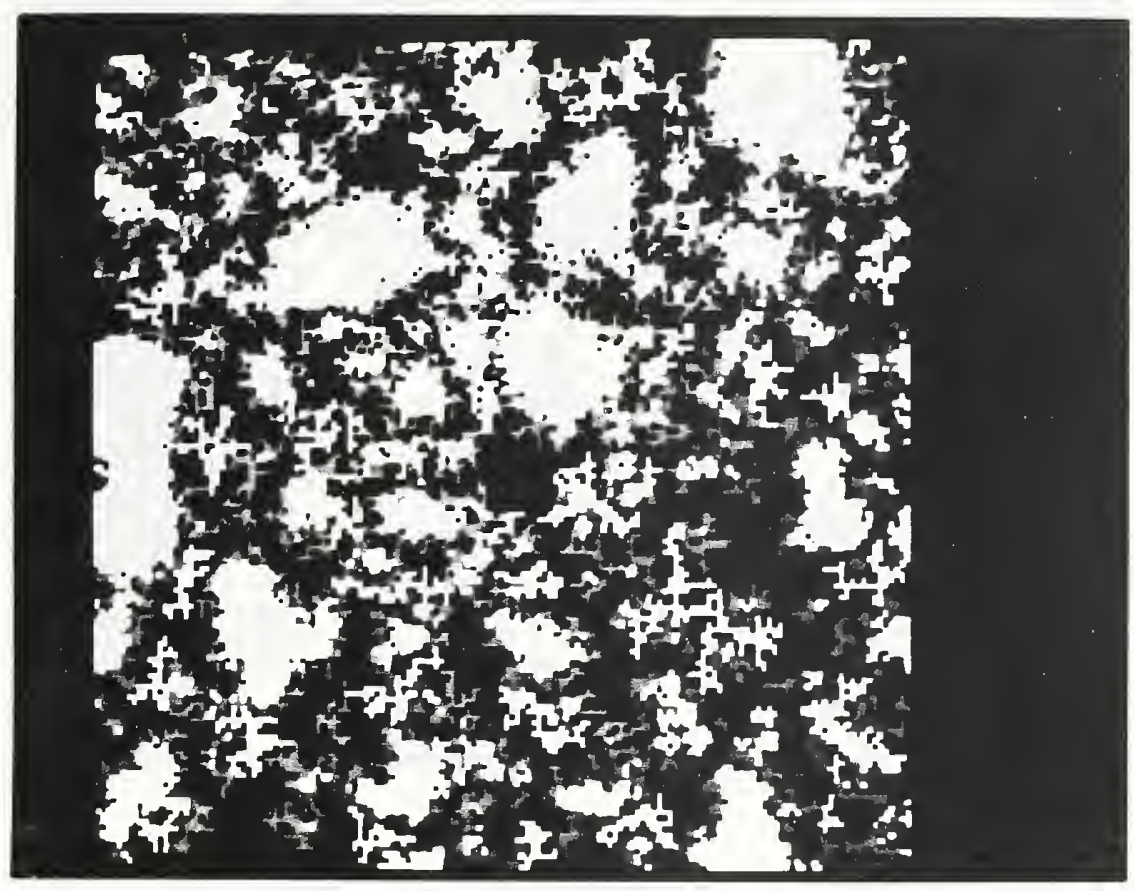

Figure 10 - 2-D Microstructure of Fig. 9 After Four Cycles of Hydration by CMMAP

The final four instructions in the file exam003.scr are utilized to execute an autocorrelation analysis of the pore structure of the image shown in Fig. 10. To obtain a working image consisting of a red pore space on a black background, the program /usr/pixar/sem/thrall3 must be executed twice, with the system user interactively providing the necessary input data. Once this working image, shown in black/white format in Fig. 11, is obtained, the program autocem is executed to obtain the raw data needed to calculate the autocorrelation function. The data file cexam03e.dat supplies the needed runtime parameters and is organized as follows:
01500150
300450100250 cexam03b.out
(coordinates of original image)
(coordinates of working image)
(file to hold intermediate results)
50
(maximum shift for which to compute autocorrelation)

When the intermediate results are analyzed by executing the program autocalc, the autocorrelation curve shown in Fig. 12 is obtained. Since the porosity in the hydrated microstructure in Fig. 10 is finely dispersed, the autocorrelation function drops rapidly to its asymptotic value. 


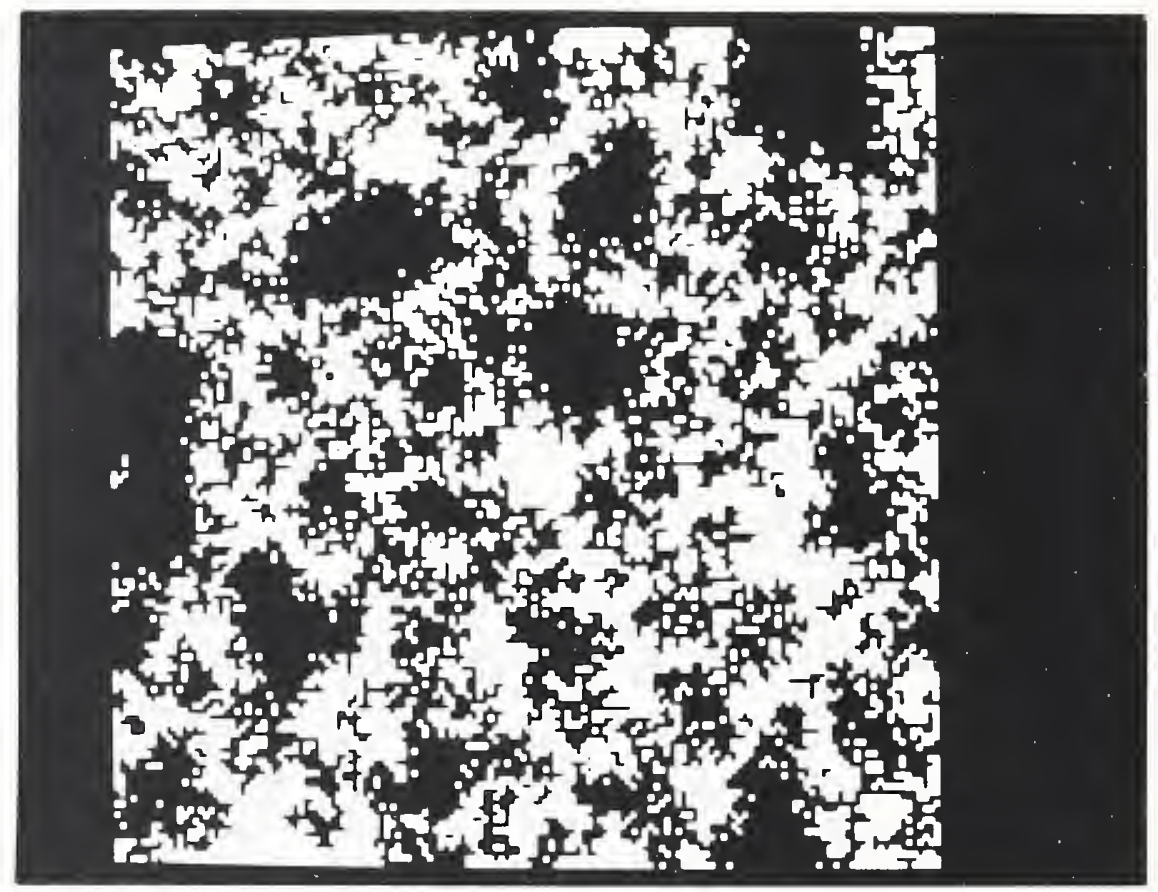

Figure 11 - Black and White Image Showing Porosity Isolated from Fig. 10

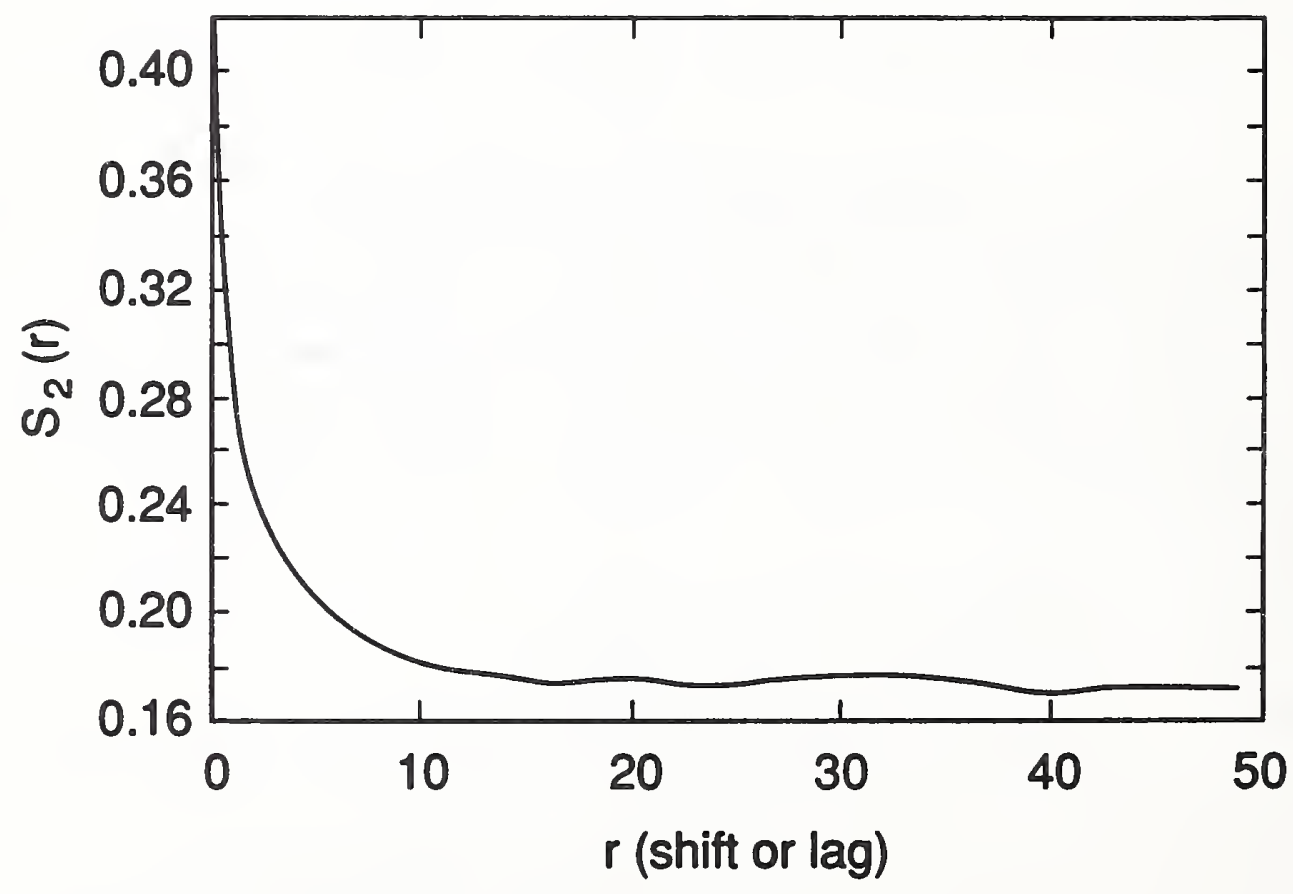

Figure 12 - Autocorrelation Curve Determined for the Porosity Shown in Fig. 11 


\section{ACKNOWLEDGEMENT}

The authors would like to thank the National Science Foundation's Science and Technology Center for Advanced Cement-Based Materials for partial support of this work. 


\section{REFERENCES}

1) "Getting Started with UNIX: Beginner's Guide, "Sun Microsystems, Mountain View, CA, 1986.

2) "UNIX: Commands Reference Manual," Sun Microsystems, Mountain View, CA, 1986.

3) "PIXAR Image Computer Programmer's Manual," PIXAR, San Rafael, CA, 1987.

4) Garboczi, E.J., "Permeability, Diffusivity, and Microstructural Parameters: A Critical Review," Cement and Concrete Research, Vol. 20, pp. 591-601, 1990.

5) Schwartz, L.M., and Banavar, J.R., Phys. Rev. B, Vol. 39, 11965, 1989.

6) Frank, D.J., and Lobb, C.J., "Highly Efficient Algorithm for Percolative Transport Studies in Two Dimensions, " Phys. Rev. B, Vol. 37, 1988.

7) Gingold, D.B., and Lobb, C.J., "Percolative Conduction in Three Dimensions," submitted to Phys. Rev. B.

8) J. van Brakel (ed.), "A Special Issue Devoted to Mercury Porosimetry," Powder Tech., Vol. 29 (1), pp. 1-209, 1981.

9) Young, J.F., "A Review of the Pore Structure of Cement Paste and Concrete and Its Influence on Permeability, " in Permeability of Concrete, ACI SP-108, Eds. Whiting D. and Walitt A., pp. 1-18, 1988.

10) Mindess, S., "Relationship Between Strength and Microstructure for Cement-Based Materials: An Overview, "in Very High Strength Cement_Based Materials, MRS Symposium Series Proceedings, Vol. 42, Ed. Young, J.F., Materials Research Society, Pittsburgh, PA., pp. 53-68, 1984.

11) Feldman, R.F., "Pore Structure, Permeability, and Diffusivity as Related to Durability," Proceedings of the 8th International Congress on the Chemistry of Cement, Rio de Janeiro, Brazil, pp. 336-346, 1986.

12) Stauffer, D., Introduction to Percolation Theory, Taylor and Francis, London, 1985.

13) Berryman, J.G., "Measurement of Spatial Correlation Functions Using Image Processing Techniques," J. Appl. Phys., Vol. 57 (7), pp. 2374-84, 1985.

14) Berrymen, J.G., and Blair, S.C., "Use of Digital Image Analysis to Estimate Fluid Permeability of Porous Materials: Application of Two-Point Correlation Functions, " J. Appl. Phys., Vol. 60 (6), pp. 1930-38, 1986. 


\section{APPENDIX A \\ START UP PROCEDURES}

To start the SUN/PIXAR system, the following steps should be performed in sequence.

1) Turn the power on for the PIXAR chassis and monitor (screen). The power switch for the PIXAR chassis is located in the read bottom left when you are facing the back of the chassis.

2) Turn the power on for the SUN workstation and monitor. The SUN will perform a series of self-tests and prompt you for a password, which may be obtained from the authors.

3) After you supply the password, the SUN will finish loading its default configuration and enter the SUNwindows environment. The default console window can be expanded and other application windows opened using the mouse as instructed in the SUN/UNIX documentation [1].

4) To use CMMAP, you must switch to the proper subdirectory by entering cd /usr/pixar/cmmap at the UNIX prompt.

5) To execute CMMAP, simply enter cmmap at the UNIX prompt. 


\section{APPENDIX B USEFUL UNIX COMMANDS}

cat filename - copy a file to the screen

cat filename $>/$ dev/ttya to send a file to the printer

cd dirname - change to a new home directory

cp file1 file2 - copy file \#1 to file \#2

fpr <infile.c > outfile.prt - program formatter; the file infile.c is formatted for printing and sent to file outfile.prt

ls dirname - display list of files located in directory dirname

mkdir dirname - create a new subdirectory called dirname

more filename - display file filename on screen one page at a time

ps - show jobs currently under execution

rm filename - delete file filename

vi filename - create/edit (full-screen) filename

where applicable $>$ - redirection of standard output

$<$ - redirection of standard input 


\section{APPENDIX C USEFUL PIXAR COMMANDS}

clr - w $x 1$ y 1 x 2 y2: clear the window specified by coordinates $x 1$ x 2 y1 y2 clr to clear the entire PIXAR screen

copy -src $\mathrm{x} 1 \mathrm{x} 2$ y1 y2 -dst $\mathrm{x} 3 \mathrm{x} 4$ y3 y4 : copy the window specified by $\mathrm{x} 1 \mathrm{x} 2$ y 1 y2 to location $\mathrm{x} 3 \mathrm{x} 4 \mathrm{y} 3 \mathrm{y} 4$

gt filename -w $x 1 \times 2$ y1 y2 : retrieve the image stored under filename from disk into the window specified by $\mathrm{x} 1 \mathrm{x} 2$ y 1 y 2

pixinit : initialize the PIXAR memory, screen, etc.

ptext $-\mathrm{t}$ words $-\mathrm{p}$ x y $-\mathrm{c} c 1 \mathrm{c2} \mathrm{c3} \mathrm{c4}$ : draw text specified by words on the screen at location $\mathrm{x}, \mathrm{y}$ in color specified by $\mathrm{c} 1 \mathrm{c} 2 \mathrm{c} 3 \mathrm{c} 4$ (for each of the four PIXAR color channels)

resize -src $\mathrm{x} 1 \mathrm{x} 2$ y 1 y 2 -dst $\mathrm{x} 3 \mathrm{x} 4$ y 3 y4 : resize the image in window specified by $\mathrm{x} 1 \mathrm{x} 2$ y 1 y 2 to fit in the window specified by $\mathrm{x} 3 \mathrm{x} 4 \mathrm{y} 3 \mathrm{y} 4$

see filename : display a raster image file stored in one of a variety of formats options are used to specify the number of bytes occupied by a header or label, the image type and resolution ( 8 or 16 bit), scaling of pixel values, etc.

sv filename - $\mathrm{x} x 1 \mathrm{x} 2 \mathrm{y} 1 \mathrm{y} 2$ : save the image located in the window specified by $\mathrm{x} 1 \mathrm{x} 2 \mathrm{y} 1$ y 2 into filename

tool : frame buffer manipulation tool (Enter ? for help) 


\section{APPENDIX D BIBLIOGRAPHY}

1) Garboczi, E.J. and Bentz, D.P., "Numerical and Analytical Models of Transport in Porous Cementitious Materials," Scientific Basis for Nuclear Waste Management, MRS Symposium Series Proceedings, Eds. Oversby, V.M. and Brown, P.W., Materials Research Society, Pittsburgh, PA, 1990.

2) Bentz, D.P. and Garboczi, E.J., "A Digitized Simulation Model for Microstructural Development," Proceedings of the NIST/ American Ceramic Society Conference on Advances in Cement-Based Materials, 1990.

3) Garboczi, E.J. and Bentz, D.P., "Digitized Simulation of Mercury Intrusion Porosimetry," Proceedings of the NIST/ American Ceramic Society Conference on Advances in CementBased Materials, 1990.

4) Bentz, D.P., Gingold, D., Garboczi, E.J., Lobb, C.J., and Jennings, H.M., "Diffusion Studies in a Computer-Based Cement Microstructural Model, " Proceedings of the NIST/ American Ceramic Society Conference on Advances in Cement-Based Materials, 1990.

5) Garboczi, E.J. and Bentz, D.P., "Digital Simulation of the Aggregate-Cement Paste Interfacial Zone in Concrete," Journal of Materials Research, in press.

6) Bentz, D.P. and Garboczi, E.J., "Digitized Direct Simulation Model of the Microstructural Development of Cement Paste, " MRS Symposium Series Proceedings, Materials Research Society, Pittsburgh, PA, 1990.

7) Garboczi, E.J. and Bentz, D.P., "Fundamental Computer Simulation Models for CementBased Materials," in Material Science of Concrete, Vol. 2, Ed. Jan P. Skalny.

8) Bentz, D.P., and Garboczi, E.J., "Percolation of Phases in a Three-Dimensional Cement Paste Microstructural Model," Cement and Concrete Research, in press. 


\section{APPENDIX E \\ EQUATIONS RELEVANT TO CMMAP}

1) Weibull distribution for cement particle radius values

Parameters: $\alpha$ - shape parameter

$\beta$ - scale parameter

$\gamma$ - location parameter

smin - minimum value for radius

smax - maximum value for radius

If $\mathrm{n}$ particles are requested, $\mathrm{n}$ ordered numbers, $\mathrm{x}_{\mathrm{i}}$, with values between 0 and 1 are generated. Then, rgen(i), the radius of particle number $i$, is given by

$$
\operatorname{rgen}(i)=\operatorname{integer}\left(0.5+\beta *\left(-\ln \left(x_{i}\right)\right)^{\frac{1}{\alpha}}+\gamma\right)
$$

where the inverse transform has been utilized to convert from the uniform, $U(0,1)$, distribution to the Weibull. Smin and smax are utilized to truncate the Weibull distribution. That is,

$$
\begin{array}{ll}
\text { if } \operatorname{rgen}(i)<\operatorname{smin} & \text { then } \operatorname{rgen}(i)=\operatorname{smin} \\
\text { if } \operatorname{rgen}(i)>\operatorname{smax} & \text { then } \operatorname{rgen}(i)=\operatorname{smax} .
\end{array}
$$

2) Nucleation probability for Calcium Hydroxide $(\mathrm{CH})$

Parameters: $\mathrm{P}_{0}$ - maximum nucleation probability

$\mathrm{N}_{\mathrm{Max}}$ - nucleation scale probability

$\mathrm{N}_{\mathrm{CH}}$ - number of $\mathrm{CH}$ diffusing species remaining

The probability $\mathrm{P}(0.0-1.0)$ of a given $\mathrm{CH}$ diffusing species nucleating at its current location in the microstructure is given by:

$$
P=P_{0} *\left(1-e^{-\left(\frac{N_{C A}}{N_{\text {Lax }}}\right)}\right.
$$


3) Equations for determination of autocorrelation function [13]

Parameters: $r$ - distance or lag

$S_{2}(r)$ - autocorrelation value at distance $r$

The autocorrelation value at distance $r$ is given by the equation

$$
S_{2}(r)=\frac{1}{2 * r+1} * \sum_{l=0}^{2 * r} S_{2}\left(r, \frac{\pi * l}{4 * r}\right)
$$

where

$$
S_{2}(r, \theta)=S_{2}(r * \cos \theta, r * \sin \theta)
$$

and $S_{2}(x, y)$ is obtained from bilinear interpolation of values of $S_{2}$ available from the image overlaying process, where for an $n$ by $m$ image

$$
S_{2}(i, j)=\frac{\sum_{k=1}^{n-i} \sum_{l=1}^{m-j} S(k, l) * S(k+i, l+j)}{(n-i) *(m-j)}
$$

and $S(k, 1)$ is 1 if the pixel at location $k, 1$ is part of the phase of interest and 0 otherwise. 


\section{APPENDIX F \\ EXAMPLE SESSION TRANSCRIPTS}

1) Transcript for Example 1

Enter script file name to be created exam001.scr

0) Exit

1) Generate a starting microstructure

2) Run a hydration model

3) Compute diffusivities

4) Perform simulated mercury intrusion

5) Compute connectivity of pore space

6) Perform general microstructural characterization

7) Perform utility functions (image manipulation, etc.)

7

0) Exit to main menu

1) Clear the PIXAR screen

2) Copy a portion of the PIXAR screen to a new location

3) Save an image to disk

4) Retrieve an image from disk

5) Remove an image from disk

6) Execute a CHAP C program

7) Execute a UNIX operating system command

1

Enter coordinates (xlo xhi ylo yhi) of window to clear Enter 0127901631 to clear the entire screen

0127901631

0) Exit to main menu

1) Clear the PIXAR screen

2) Copy a portion of the PIXAR screen to a new location

3) Save an image to disk

4) Retrieve an image from disk

5) Remove an image from disk

6) Execute a CHAP C program

7) Execute a UNIX operating system command

0 
0) Exit

1) Generate a starting microstructure

2) Run a hydration model

3) Compute diffusivities

4) Perform simulated mercury intrusion

5) Compute connectivity of pore space

6) Perform general microstructural characterization

7) Perform utility functions (image manipulation, etc.)

1

0) Exit to main menu

1) Create a 2-D microstructure using circles of discrete sizes

2) Create a 2-D microstructure using circles of a specified size distribution

3) Create a 2-D microstructure using cement particle shapes stored in a database

4) Retrieve a 2-D microstructure from disk

5) Create a 3-D microstructure using spheres of discrete sizes

6) Create a 3-D microstructure using spheres of a specified size distribution

7) Retrieve a 3-D microstructure from disk

1

Enter filename to be established to hold parameter values cexam01.dat

Enter number of circles to generate

240

Enter diameter (in pixels) of circles to be generated

21

Enter number of random numbers to generate at start

0

Enter coordinates for box to place circles in (xlo xhi ylo yhi)

05000500

0) Exit to main menu

1) Create a 2-D microstructure using circles of discrete sizes

2) Create a 2-D microstructure using circles of a specified size distribution

3) Create a 2-D microstructure using cement particle shapes stored in a database

4) Retrieve a 2-D microstructure from disk

5) Create a 3-D microstructure using spheres of discrete sizes

6) Create a 3-D microstructure using spheres of a specified size distribution

7) Retrieve a 3-D microstructure from disk

0 
0) Exit

1) Generate a starting microstructure

2) Run a hydration model

3) Compute diffusivities

4) Perform simulated mercury intrusion

5) Compute connectivity of pore space

6) Perform general microstructural characterization

7) Perform utility functions (image manipulation, etc.)

5

0) Exit to main menu

1) Compute 2-D connectivity of pore space in 2-D microstructure

2) Compute 2-D connectivity of pore space in 3-D microstructure

3) Compute 3-D connectivity of pore space in 3-D microstructure 1

Enter filename to be established to hold parameters for analysis cexam01a.dat

Enter coordinates (xlo xhi ylo yhi) for image box

05000500

Enter coordinates for temporary storage window \#1

60010990499

Enter coordinates for temporary storage window \#2

6001099500999

Do you wish to send output to 0 ) the screen or 1) a file

0

0) Exit to main menu

1) Compute 2-D connectivity of pore space in 2-D microstructure

2) Compute 2-D connectivity of pore space in 3-D microstructure

3) Compute 3-D connectivity of pore space in 3-D microstructure 0 
0) Exit

1) Generate a starting microstructure

2) Run a hydration model

3) Compute diffusivities

4) Perform simulated mercury intrusion

5) Compute connectivity of pore space

6) Perform general microstructural characterization

7) Perform utility functions (image manipulation, etc.)

4

0) Exit to main menu

1) Perform 2-D mercury intrusion

2) Perform 3-D mercury intrusion

1

Enter filename to be established to hold mercury intrusion parameters cexam01b.dat

Enter coordinates (xlo xhi ylo yhi) for storage window \#1

0499600799

Enter coordinates for storage window \#2

0499800999

Enter coordinates of original image to be intruded

05000500

Enter coordinates of working image for intrusion(same size as original) 6001100100600

Enter minimum and maximum radii for intrusion process

112

Do you wish to output results to 0 ) the screen or 1 ) a file 1

Enter filename to send output results to mexam01.out

0) Exit to main menu

1) Perform 2-D mercury intrusion

2) Perform 3-D mercury intrusion

0 

0) Exit
1) Generate a starting microstructure
2) Run a hydration model
3) Compute diffusivities
4) Perform simulated mercury intrusion
5) Compute connectivity of pore space
6) Perform general microstructural characterization
7) Perform utility functions (image manipulation, etc.)
0

Thank you for using CMMAP

To execute your analysis, please type

csh exam001.scr at the UNIX prompt 
2) Transcript for Example 2

Enter script file name to be created exam002.sct

0) Exit

1) Generate a starting microstructure

2) Run a hydration model

3) Compute diffusivities

4) Perform simulated mercury intrusion

5) Compute connectivity of pore space

6) Perform general microstructural characterization

7) Perform utility functions (image manipulation, etc.)

7

0) Exit to main menu

1) Clear the PIXAR screen

2) Copy a portion of the PIXAR screen to a new location

3) Save an image to disk

4) Retrieve an image from disk

5) Remove an image from disk

6) Execute a CHAP C program

7) Execute a UNIX operating system command

1

Enter coordinates (xlo xhi ylo yhi) of window to clear Enter 0127901631 to clear the entire screen

0127901631

0) Exit to main menu

1) Clear the PIXAR screen

2) Copy a portion of the PIXAR screen to a new location

3) Save an image to disk

4) Retrieve an image from disk

5) Remove an image from disk

6) Execute a CHAP C program

7) Execute a UNIX operating system command

0 
0) Exit

1) Generate a starting microstructure

2) Run a hydration model

3) Compute diffusivities

4) Perform simulated mercury intrusion

5) Compute connectivity of pore space

6) Perform general microstructural characterization

7) Perform utility functions (image manipulation, etc.)

0) Exit to main menu

1) Create a 2-D microstructure using circles of discrete sizes

2) Create a 2-D microstructure using circles of a specified size distribution

3) Create a 2-D microstructure using cement particle shapes stored in a database

4) Retrieve a 2-D microstructure from disk

5) Create a 3-D microstructure using spheres of discrete sizes

6) Create a 3-D microstructure using spheres of a specified size distribution

7) Retrieve a 3-D microstructure from disk

6

Enter filename to be established to hold parameter values cexam02a.dat

Enter number of spheres to be generated

500

Enter alpha parameter for Weibull distribution

2.0

Enter beta parameter for Weibull distribution

4.0

Enter gamma parameter for Weibull distribution

1.0

Enter minimum and maximum radii to be used for spheres

19

Enter maximum number of random attempts to be made in placing spheres

500 
0) Exit to main menu

1) Create a 2-D microstructure using circles of discrete sizes

2) Create a 2-D microstructure using circles of a specified size distribution

3) Create a 2-D microstructure using cement particle shapes stored in a database

4) Retrieve a 2-D microstructure from disk

5) Create a 3-D microstructure using spheres of discrete sizes

6) Create a 3-D microstructure using spheres of a specified size distribution

7) Retrieve a 3-D microstructure from disk

0

0) Exit

1) Generate a starting microstructure

2) Run a hydration model

3) Compute diffusivities

4) Perform simulated mercury intrusion

5) Compute connectivity of pore space

6) Perform general microstructural characterization

7) Perform utility functions (image manipulation, etc.)

5

0) Exit to main menu

1) Compute 2-D connectivity of pore space in 2-D microstructure

2) Compute 2-D connectivity of pore space in 3-D microstructure

3) Compute 3-D connectivity of pore space in 3-D microstructure 3

Do you wish to send the results to 0 ) the screen or 1) a file 1 Enter filename to write results to cexam02a.out

0) Exit to main menu

1) Compute 2-D connectivity of pore space in 2-D microstructure

2) Compute 2-D connectivity of pore space in 3-D microstructure

3) Compute 3-D connectivity of pore space in 3-D microstructure 0 

0) Exit
1) Generate a starting microstructure
2) Run a hydration model
3) Compute diffusivities
4) Perform simulated mercury intrusion
5) Compute connectivity of pore space
6) Perform general microstructural characterization
7) Perform utility functions (image manipulation, etc.)
3

0) Exit to main menu

1) Compute 2-D diffusivity using blind ant algorithm

2) Compute 2-D diffusivity using y-del algorithm

3) Compute 3-D diffusivity using blind ant algorithm

3

Enter filename to be established to hold ant run time parameters cexam02b.dat

Enter number of ants to use to compute diffusivity

10000

Enter number of steps for each ant to take

100000

0) Exit to main menu

1) Compute 2-D diffusivity using blind ant algorithm

2) Compute 2-D diffusivity using $y$-del algorithm

3) Compute 3-D diffusivity using blind ant algorithm

0

0) Exit

1) Generate a starting microstructure

2) Run a hydration model

3) Compute diffusivities

4) Perform simulated mercury intrusion

5) Compute connectivity of pore space

6) Perform general microstructural characterization

7) Perform utility functions (image manipulation, etc.)

2 
0) Exit to main menu

1) Execute the 2-D hydration model

2) Execute the 3-D hydration model

2

Enter filename to be established to hold hydration parameters cexam02c.dat

Enter number of cycles of hydration to perform

20

Enter maximum number of diffusion steps per cycle

10000

Enter maximum probability $(0.0-1.0)$ for $\mathrm{CH}$ nucleation

0.001

Enter exponential scale factor for $\mathrm{CH}$ nucleation

100000 .

Do you wish the output to go to 0 ) the screen or 1) a file 1

Enter filename to send output results to cexam02b.out

0) Exit to main menu

1) Execute the 2-D hydration model

2) Execute the 3-D hydration model

0

0) Exit

1) Generate a starting microstructure

2) Run a hydration model

3) Compute diffusivities

4) Perform simulated mercury intrusion

5) Compute connectivity of pore space

6) Perform general microstructural characterization

7) Perform utility functions (image manipulation, etc.)

5

0) Exit to main menu

1) Compute 2-D connectivity of pore space in 2-D microstructure

2) Compute 2-D connectivity of pore space in 3-D microstructure

3) Compute 3-D connectivity of pore space in 3-D microstructure 3 
Do you wish to send the results to 0 ) the screen or 1) a file 1

Enter filename to write results to cexam02c.out

0) Exit to main menu

1) Compute 2-D connectivity of pore space in 2-D microstructure

2) Compute 2-D connectivity of pore space in 3-D microstructure

3) Compute 3-D connectivity of pore space in 3-D microstructure

0

0) Exit

1) Generate a starting microstructure

2) Run a hydration model

3) Compute diffusivities

4) Perform simulated mercury intrusion

5) Compute connectivity of pore space

6) Perform general microstructural characterization

7) Perform utility functions (image manipulation, etc.)

0

Thank you for using CMMAP

To execute your analysis, please type

csh exam002.scr at the UNIX prompt 


\section{3) Transcript for Example 3}

Enter script file name to be created exam003.scr

0) Exit

1) Generate a starting microstructure

2) Run a hydration model

3) Compute diffusivities

4) Perform simulated mercury intrusion

5) Compute connectivity of pore space

6) Perform general microstructural characterization

7) Perform utility functions (image manipulation, etc.)

7

0) Exit to main menu

1) Clear the PIXAR screen

2) Copy a portion of the PIXAR screen to a new location

3) Save an image to disk

4) Retrieve an image from disk

5) Remove an image from disk

6) Execute a CHAP C program

7) Execute a UNIX operating system command

1

Enter coordinates (xlo xhi ylo yhi) of window to clear Enter 0127901631 to clear the entire screen 0127901631

0) Exit to main menu

1) Clear the PIXAR screen

2) Copy a portion of the PIXAR screen to a new location

3) Save an image to disk

4) Retrieve an image from disk

5) Remove an image from disk

6) Execute a CHAP C program

7) Execute a UNIX operating system command 0 

0) Exit
1) Generate a starting microstructure
2) Run a hydration model
3) Compute diffusivities
4) Perform simulated mercury intrusion
5) Compute connectivity of pore space
6) Perform general microstructural characterization
7) Perform utility functions (image manipulation, etc.)
1

0) Exit to main menu

1) Create a 2-D microstructure using circles of discrete sizes

2) Create a 2-D microstructure using circles of a specified size distribution

3) Create a 2-D microstructure using cement particle shapes stored in a database

4) Retrieve a 2-D microstructure from disk

5) Create a 3-D microstructure using spheres of discrete sizes

6) Create a 3-D microstructure using spheres of a specified size distribution

7) Retrieve a 3-D microstructure from disk

3

Enter filename to be established to hold parameter values cexam03a.dat

Enter number of particles to be placed in box

264

Enter coordinates for box to place circles in (xlo xhi ylo yhi)

01500150

Enter number of random numbers to generate prior to placement

0

0) Exit to main menu

1) Create a 2-D microstructure using circles of discrete sizes

2) Create a 2-D microstructure using circles of a specified size distribution

3) Create a 2-D microstructure using cement particle shapes stored in a database

4) Retrieve a 2-D microstructure from disk

5) Create a 3-D microstructure using spheres of discrete sizes

6) Create a 3-D microstructure using spheres of a specified size distribution

7) Retrieve a 3-D microstructure from disk

0 

0) Exit
1) Generate a starting microstructure
2) Run a hydration model
3) Compute diffusivities
4) Perform simulated mercury intrusion
5) Compute connectivity of pore space
6) Perform general microstructural characterization
7) Perform utility functions (image manipulation, etc.)
3

0) Exit to main menu

1) Compute 2-D diffusivity using blind ant algorithm

2) Compute 2-D diffusivity using y-del algorithm

3) Compute 3-D diffusivity using blind ant algorithm

2

Enter filename to be established to hold y-del run parameters cexam03b.dat

Enter coordinates (xlo xhi ylo yhi) for original image window 01500150

Enter coordinates for horizontal bonds window

200360100260

Enter coordinates for vertical bonds window 200360300460

0) Exit to main menu

1) Compute 2-D diffusivity using blind ant algorithm

2) Compute 2-D diffusivity using y-del algorithm

3) Compute 3-D diffusivity using blind ant algorithm

0

0) Exit

1) Generate a starting microstructure

2) Run a hydration model

3) Compute diffusivities

4) Perform simulated mercury intrusion

5) Compute connectivity of pore space

6) Perform general microstructural characterization

7) Perform utility functions (image manipulation, etc.)

2 
0) Exit to main menu

1) Execute the 2-D hydration model

2) Execute the 3-D hydration model

1

Enter the filename to be created to hold the hydration parameters cexam03c.dat

Do you wish to perform expansion (CSH) by

1) generating extra ions during each dissolution

2) probabilistic-based expansion of species upon reaction 1

Do you wish to utilize

1) periodic boundaries or

2) reflecting walls

2

Enter maximum probability $(0.0-1.0)$ for $\mathrm{CH}$ nucleation 0.005

Enter exponential scale factor for $\mathrm{CH}$ nucleation

50000.

Enter sticking probability for $\mathrm{CSH}$ to $\mathrm{C}_{3} \mathrm{~S}(0.0-1.0)$

1.0

Enter sticking probability for CSH to $\mathrm{CSH}(0.0-1.0)$

1.0

Enter number of hydration cycles to perform

4

Enter maximum number of diffusion steps per cycle

10000

Enter coordinates (xlo xhi ylo yhi) for hydration window

01500150

Enter coordinates for ion location window number one

5009990499

Enter coordinates for ion location window number two

500999500999

Do you wish to 0 ) send output to screen or 1) send output to a file 1

Enter filename to send results to

cexam03a.out

0) Exit to main menu

1) Execute the 2-D hydration model

2) Execute the 3-D hydration model

0 

0) Exit
1) Generate a starting microstructure
2) Run a hydration model
3) Compute diffusivities
4) Perform simulated mercury intrusion
5) Compute connectivity of pore space
6) Perform general microstructural characterization
7) Perform utility functions (image manipulation, etc.)
3

0) Exit to main menu

1) Compute 2-D diffusivity using blind ant algorithm

2) Compute 2-D diffusivity using $y$-del algorithm

3) Compute 3-D diffusivity using blind ant algorithm

2

Enter filename to be established to hold $y$-del run parameters cexam03d.dat

Enter coordinates (xlo xhi ylo yhi) for original image window 01500150 Enter coordinates for horizontal bonds window 200360100260

Enter coordinates for vertical bonds window 200360300460

0) Exit to main menu

1) Compute 2-D diffusivity using blind ant algorithm

2) Compute 2-D diffusivity using $y$-del algorithm

3) Compute 3-D diffusivity using blind ant algorithm 0

0) Exit

1) Generate a starting microstructure

2) Run a hydration model

3) Compute diffusivities

4) Perform simulated mercury intrusion

5) Compute connectivity of pore space

6) Perform general microstructural characterization

7) Perform utility functions (image manipulation, etc.) 
0) Exit to main menu

1) Clear the PIXAR screen

2) Copy a portion of the PIXAR screen to a new location

3) Save an image to disk

4) Retrieve an image from disk

5) Remove an image from disk

6) Execute a CHAP C program

7) Execute a UNIX operating system command

6

Enter filename of program to execute

/usr/pixar/sem/thrall3

0) Exit to main menu

1) Clear the PIXAR screen

2) Copy a portion of the PIXAR screen to a new location

3) Save an image to disk

4) Retrieve an image from disk

5) Remove an image from disk

6) Execute a CHAP C program

7) Execute a UNIX operating system command

6

Enter filename of program to execute

/usr/pixar/sem/thrall3

0) Exit to main menu

1) Clear the PIXAR screen

2) Copy a portion of the PIXAR screen to a new location

3) Save an image to disk

4) Retrieve an image from disk

5) Remove an image from disk

6) Execute a CHAP C program

7) Execute a UNIX operating system command

0 
0) Exit

1) Generate a starting microstructure

2) Run a hydration model

3) Compute diffusivities

4) Perform simulated mercury intrusion

5) Compute connectivity of pore space

6) Perform general microstructural characterization

7) Perform utility functions (image manipulation, etc.)

6

0) Exit to main menu

1) Characterize discrete objects by size, location, etc.

2) Characterize complex phases by autocorrelation analysis

2

Enter filename to hold runtime parameters for autocorrelation cexam03e.dat

Enter coordinates (xlo xhi ylo yhi) of image to be processed

01500150

Enter coordinates to hold shifted image

300450100250

Enter filename to write results to

cexam03b.out

Enter maximum lag to be used in determining autocorrelation

50

0) Exit to main menu

1) Characterize discrete objects by size, location, etc.

2) Characterize complex phases by autocorrelation analysis

0

0) Exit

1) Generate a starting microstructure

2) Run a hydration model

3) Compute diffusivities

4) Perform simulated mercury intrusion

5) Compute connectivity of pore space

6) Perform general microstructural characterization

7) Perform utility functions (image manipulation, etc.)

7 

0) Exit to main menu
1) Clear the PIXAR screen
2) Copy a portion of the PIXAR screen to a new location
3) Save an image to disk
4) Retrieve an image from disk
5) Remove an image from disk
6) Execute a CHAP C program
7) Execute a UNIX operating system command
7

Enter UNIX command to execute autocalc

0) Exit to main menu

1) Clear the PIXAR screen

2) Copy a portion of the PIXAR screen to a new location

3) Save an image to disk

4) Retrieve an image from disk

5) Remove an image from disk

6) Execute a CHAP C program

7) Execute a UNIX operating system command 0

0) Exit

1) Generate a starting microstructure

2) Run a hydration model

3) Compute diffusivities

4) Perform simulated mercury intrusion

5) Compute connectivity of pore space

6) Perform general microstructural characterization

7) Perform utility functions (image manipulation, etc.)

0

Thank you for you using CMMAP

To execute your analysis, please type

csh exam003.scr

at the UNIX prompt 



\section{BIBLIOGRAPHIC DATA SHEET}

2. PERForming ORGAMIZATION REPORT NUMBEA

3. PUBLICATION DATE

OCTOBER 1990

1. TITLEAND SUBTITLE

User's Guide to CMMAP: Cement Microstructure Modelling and Analysis Package

5. AUTHOR(S)

Dale P. Bentz and Edward J. Garboczi

6. PERFORMING ORGANIZATION (IF JOINT OR OTHER THAN MIST, SEE INSTRUCTIONS)

U.S. DEPARTMENT OF COMMERCE

NATIONAL INSTITUTE OF STANDARDS AND TECHNOLOQY

GAITHERSBUAG, MD 20899

7. CONTRACT/GRANT MUMBER

8. TYPE OF REPORT AND PEAIOD COVERED

9. SPONSORING ORGANIZATION NAME AND COMPLETE ADDRESS (STREET, CITY, STATE, ZIP)

A collection of modelling and analysis programs dealing with cement microstructure has been assembled into a single package. This manual describes the custom user interface, CMMAP, which allows the user to access and execute the assembled software. Specific programs exist to create starting microstructures, simulate hydration, and analyze both starting and hydrated microstructures. Available analyses include evaluation of diffusion coefficients, simulation of mercury intrusion porosimetry, and assessment of the connectivity of phases within a cement microstructure. The menu-based interface program is described in detail and several examples are presented which demonstrate the capabilities of CMMAP.

12. KEY WOADS (6 TO 12 ENTRIES; ALPHABETICAL ORDER; CAPITALZE ONLY PROPER NAMES; AND SEPARATE KEY WORDS BY SEMICOLONS) Cement; computer imaging; connectivity; diffusion; hydration; microstructure; modelling; simulation

FOR OFFICLAL DISTRIBUTION. DO NOT RELEASE TO MATIONAL TECHMICAL INFORMATION SERVCE (NTIS).

14. NUMBER OF PRINTED PAGES

5.7

15. PRICE

$\mathrm{A} 04$ 


